\title{
IL CAMPO GEOMAGNETICO
}

\author{
FRANCO MOLINA
}

Dirigente il reparto geomagnetico dell'Istituto Nazionale di Geofisica 


\section{PREFAZIONL}

Questo lavoro non è un trattato sul campo geomagnetico e nemmeno una rassegna sull'argomento, almeno nel significato che normalmente si attribuisce al termine "rassegna "; esso è semplicemente un tentativo di condensare in termini comprensibili agli studiosi non specializzati ciò che oggi si sa o si ipotizza sul magnetismo terrestre e sulle sue cause. Chi scrive si è sempre trovato a disagio di fronte alle richieste di una spiegazione sull'origine del campo magnetico terrestre: o si cerca di cavarsela con poche parole (" si tratta di correnti elettriche indotte nell'interno della Terra") che possono forse accontentare chi non si occupa in modo particolare di problemi scientifici ma non possono soddisfare un fisico, oppure ci si sente obbligati a tentare faticosamente di approfondire, sia per capirli personalmente che per poterli poi spiegare agli altri, argomenti e ragionamenti che sono in realtà accessibili solo a un fisico teorico ben preparato. Apparentemente non esiste una via di mezzo, come del resto in molti altri campi della fisica contemporanea.

La presente monografia rappresenta appunto il tentativo di trovare questa via di mezzo: nel terzo capitolo, dedicato alle teorie sull'origine del campo geomagnetico, si cerca di descrivere qualitativamente i principali processi fisici proposti come sorgenti del campo, dopo un accenno ai fondamenti fisici e matematici sui quali sono state elaborate le varie teorie. Senza farmi soverchie illusioni di essere riuscito nell'intento, mi dichiarerei tuttavia soddisfatto se questo lavoro destasse sull'argomento l'interesse di qualche giovane ricercatore, incoraggiandolo ad un approfondimento.

I primi due capitoli sono introduttivi al terzo ora citato, e illustrano i dati sperimentali necessari a chi intende cercare le cause del campo geomagnetico. In particolare il secondo capitolo, dedicato al paleomagnetismo, oltre a presentare un compendio di dati sulla storia del campo, ha anche come mira di attirare l'attenzione degli studiosi di fisica e di geologia su di un campo di ricerca suscettibile di varie e interessanti applicazioni scientifiche; per la natura di questo lavoro, 
in esso non sono stati tuttavia trattati i fondamenti della elaborazione statistica dei dati sperimentali e della teoria degli errori relativi ai metorli di misurazione. Inoltre, per la comprensione degli argomenti trattati in questo capitolo è necessaria la conoscenza dei fonclamenti del magnetismo nella materia; per non dilungarsi eccessivamente, essi vengono qui supposti noti. In ogni caso, rlelucirlazioni piuttosto esaurienti si potranno ottenere clai rlue trattati di Nagata e di Irving citati nella bibliografia.

A proposito di questa, dato appunto che il presente lavoro non è una rassegna, essa i stata ridotta a poche voci, inclurlenti alcuni lavori generali sugli argomenti trattati e alcune rlelle piu significative note scientifiche; una bibliografia più completa si può trovare nei lavori citati. Ritengo qui opportuno prevenire il lettore rlesirleroso di approfondire che, mentre per le teorie sull origine del campo la letteratura non è abbondantissima e può essere da questo punto di vista abbastanza facilmente affrontata, nel campo del paleomagnetismo il numero rli lavori pubblicati è rlivenuto enorme; sarebbe quindi vivamente augurabile un aggiornamento dei trattati generali qui citati.

Roma, I Dicembre 1972 . 


\section{INTROJUUIONE}

Nel trattare l'argomento oggetto del presente lavoro si pone naturalmente il problema del sistema di unità da usare per le grandezze fisiche e per le equazioni. I'Unione Internazionale di Fisica pura e applicata (I.U.P.A.P.) ha ufficialmente raceomandato l'uso universale del Sistema Internazionale (S.I.), che, come si sa, per l'elettromagnetismo ì il sistema MIKSA. Come è noto, in Geomagnetismo ì stato sempre usato, almeno per esprimere le granclezze fisiche, il sistema CGS elettromagnetico (u.e.m.); le proposte avanzate in seno alla Associazione Internazionale di Geomagnetismo e Aeronomia (I.A.G.A.), durante il congresso di MLosea (1971) dell'Unione Geofisica e Geodetica Internazionale, per l'arlozione del sistema S.I. in Geomagnetismo non sono state accolte (probabilmente per ragioni più formali che sostanziali), ma è stata lasciata la porta aperta per un'accettazione futura; è quincli scontato che in un tempo più o meno prossimo si giungerà all'uso del sistema S.I. in tutti i rami della Geofisica.

Data questa situazione, che possiamo chiamare di attesa se non proprio di transizione, si è ritenuto opportuno in questo lavoro esprimere le grandezze fisiche nel sistema u.e.m., dato che esso è il sistema tradizionalmente adottato (tanto tradizionalmente che il suo uso può considerarsi ufficiale), aggiungendo fra parentesi il loro valore nel sistema S.I., che sarà certamente il sistema di clomani. Questa soluzione, indubbiamente poco gradevole alla lettura, permetterà tuttavia al lettore che vorrà approfondire i vari argomenti di orientarsi più facilmente quando consulterà direttamente le pubblicazioni scientifiche passate e quelle future. Eे ovvio che tale doppia inclicazione non sarà usata per le grandezze semplici (come ad es. la velocità), che saranno senz'altro espresse in S.I.; inoltre, tutte le equazioni che compaiono nel Cap. 3, dove vengono accennate le teorie sull'origine del campo geomagnetico, sono scritte esclusivamente nella forma che deriva dall'uso del sistema S.I.

Le grandezze magnetiche usate in questo lavoro e soggette alla doppia unità di misura sono quattro: il "campo " magnetico, il mo- 
mento magnetico, l'intensità di magnetizzazione (cioè il momento magnetico per unità di volume) e la suscettività. La loro definizione comporta delle difficoltà che sono almeno in parte di natura diversa da quelle derivanti da una semplice trasformazione di unità.

Per quanto riguarda il " campo ", l'introduzione in fisica (dettata dalla necessità di descrivere adeguatamente i fenomeni magnetici all'interno della materia) di due vettori diversi, $\boldsymbol{H}$, intensità del campo, e $\boldsymbol{B}$, induzione magnetica, rende necessario decidere che cosa effettivamente si misuri con i vari strumenti ideati per la rleterminazione del campo geomagnetico: seconclo la fisica tradizionale, un ago (dipolo) magnetico nelle sue diverse applicazioni (metodo di GaussLamont, galvanometro dei seni ecc.) conduce alla misura di $H$, mentre una bobina di induzione, come quelle usate per le micropulsazioni, o un "search-coil " usato sui satelliti, che non è altro che un semplicissimo induttore terrestre, misurano $\mathrm{l} B / \mathrm{d} t$. L'uso del sistema u.e.m. ha sempre permesso di sorvolare su questa complicazione, in quanto in esso $H$ e $B$ non solo hanno le stesse dimensioni fisiche, ma nel vuoto, e praticamente nell'aria, hanno anche lo stesso valore numerico; si sono quindi usate indifferentemente le unità oersted e gauss (con la preferenza alla seconda in passato, alla prima negli anni più recenti), con l'uso universale del gamma $(\gamma)$, definito a sua volta indifferentemente come $10^{-5}$ oersted o $10^{-5}$ gauss. Nel sistema S.I. è invece necessario distinguere fra le unità di $H$, l'Ampere/metro $\left(A \mathrm{~m}^{-1}\right)$, e l'unità di $B$, il Weber $/ \mathrm{m}^{2}$, detto ora tesla (T); $H$ ha le rimensioni $[H]=$ $\left[I L^{-1}\right](I \equiv$ intensità di corrente $)$, mentre per $B$ si ha $[B]=\left[M L t^{-2} / I L\right]=$ $\left[M t^{-}-I^{-1}\right]$. La relazione tra $B$ e $H$ ̀̀ $B=\mu H$, dove $\mu$ è la permeabilità magnetica, che ha le dimensioni di una forza diviso per il quarrato di una intensità di corrente $\left([\mu]=\left[M L t^{-2} I^{-2}\right]\right)$; nel vuoto, e praticamente nell'aria, si ha $\boldsymbol{B}_{o}=\mu_{0} \boldsymbol{H}_{o}$, dove $\mu_{0}$ ha le stesse dimensioni di $\mu$ e il valore numerico $4 \pi \cdot 10^{-7}$. Le relazioni fra le grandezze del sistema S.I. e le corrispondenti grandezze del sistema u.e.m. sono:

$$
1 \mathrm{~A} / \mathrm{m}=4 \pi \cdot 10^{-3} \text { oersted; } 1 \mathrm{~T}=10^{4} \text { gauss. }
$$

La situazione è cambiata in seguito a una recente decisione della I.U.P.A.P. sulla definizione di momento magnetico. Non potendosi dare del momento magnetico una definizione analoga a quella del momento elettrico, a causa della impossibilità di individuare la " massa magnetica " come analogo della carica elettrica, lo si è definito come quella grandezza vettoriale che lega fra loro l'energia potenziale $E$ 
di un dipolo magnetico in un eampo uniforme e il vettore di campo, che indicheremo genericamente con $F$ :

$$
E=-M \times F \text {. }
$$

Esistono due possibilitì di scelta per $F$, e precisamente $H$ oppure $B$. Se si sceglie $H$, come è stato fatto in passato, il momento magnetico $M$ ha le dimensioni, nel sistema S.I.,

$$
[M]=\left[M L^{2-t^{-2}} / I L^{-1}\right]=\left[M L^{3} t^{-2} I^{-1}\right] .
$$

Se si sceglie $B$, le dimensioni di $M$ sono

$$
[M]=\left[M L^{2} t^{-2} / M t^{-2} I^{-1}\right]=[L-I] .
$$

Come si vede dalle equazioni dimensionali, la prima scelta conduce ad assumere per il momento magnetico di una spira circolare di area $S$ percorsa dalla corrente $i$ la grandezza $\mu_{o} i S$, la seconda scelta porta all un momento pari a $i s$, come nel sistema u.e.m.

Orbene, la I.U.P.A.P. ha deciso per la seconda alternativa: il momento magnetico si misura allora, nel sistema S.I., in Ampere moltiplicato $\mathrm{m}^{2}\left(\mathrm{Am}^{2}\right)$. Il significato di questa scelta è evillente: il vettore atto a descrivere il campo magnetico è solo $B$, perché ad esso viene attribuita l'azione che in precedenza caratterizzava e serviva a definire il vettore $H$, ciò̀ la coppia esercitata su di un dipolo magnetico.

Questa scelta è indubbiamente opinabile; essa tuttavia toglie ogni ambiguità dalle misure di campo magnetico nel vuoto. Una sua conseguenza sul Geomagnetismo è che le unità di misura del campo geomagnetico sono quelle di $B$ : il gauss nel sistema u.e.m., il tesla nel sistema S.I. Dalla relazione fra tesla e gauss e fra gauss e gamma risulta che $1 \gamma=10^{-9} \mathrm{~T}=1 \mathrm{n} \mathrm{T}$ (nanotesla); ciò permette di conservare l'antica unità gamma con la nuova denominazione di nT, evitando la necessità di trasformare tutti i valori numerici relativi al campo magnetico terrestre finora usati.

Quanto al momento magnetico, il fatto che esso abbia in amberue i sistemi le dimensioni di una intensità di corrente per una superficie permette di ricavare facilmente il rapporto fra le unità: 1 u.e.m. = $10^{1} \cdot 10^{-4}=10^{-3} \mathrm{Am}^{2}$.

Dalle considerazioni sul campo e sul momento si puó precisare l'espressione del campo prodotto da un dipolo di momento $M$ in un punto a distanza $r$ dal suo centro. E noto che questo i proporzionale a $M / r^{3}$; la costante di proporzionalità dipende dal sistema di unità 
usato. $\Lambda$ parte un fattore in cui compare la "latitudine " del punto, fattore comune a tutti i sistemi, la costante è uguale a 1 nel sistema u.e.m.; dato che il campo è misurato in gauss, rispettivamente in tesla, nei sistemi u.e.m. e S.I., tenendo conto dei rapporti fra le corrispondenti unità per il campo, il momento magnetico e la lunghezza nei due sistemi, si trova che in S.I. la costante è uguale a $10^{-7}$, ciò a $\mu_{o} / 4 \pi$. Questo risultato si potrebbe ritrovare anche partendo dall'equivalenza fra un dipolo e una spira circolare percorsa da corrente $(M=i S)$ e applicando la prima formula di Taplace espressa in S.I.

I)al momento magnetico si deduce immerliatamente l'intensità di magnetizzazione $I$, dividendo per un volume; nel sistema S.I. essa risulta allora misurata in $\mathrm{Am}^{-1}$, e la relazione fra le due unità è

$$
1 \frac{\text { ll.e.m. }}{\mathrm{cm}^{3}}=\frac{10^{-3}}{10^{-6}} \mathrm{Am}^{-1}=10^{3} \mathrm{Am}^{-1} .
$$

Rimane infine la suscettivitia $\chi$; volendo conservarle anche nel sistema S.I. la sua caratteristica tradizionale di grandezza adimensionata, è necessario che essa venga considerata come la grandezza che lega l'intensità di magnetizzazione $\left(\Lambda \mathrm{m}^{-1}\right)$ con un altro vettore la cui intensità abbia le dimensioni $A \mathrm{~m}^{-1}$, cioè $H$. Si avrà ciò̀ ancora la relazione $I=\chi H$. La relazione fra le due unità di $\chi$ c̀:

$$
\chi \text { u.e.m. }=\frac{1 \text { u.e.m. } / \mathrm{cm}^{3}}{1 \text { oersted }}=\frac{10^{3} \mathrm{Am}^{-1}}{10^{3} / 4 \pi \mathrm{Am}^{-1}}=4 \pi \mathrm{s} . \mathrm{L} \text {. }
$$

Va da sè che anche nel sistema u.e.m. $\chi$ è adimensionata, in quanto $I$, essendo data da un momento magnetico diviso un volume $\left(L^{3}\right)$, ha le stesse dimensioni di $H$, che è dato anch'esso da un momento magnetico diviso il cubo di una lunghezza. Questa definizione di suscettività desta tuttavia delle perplessità: se da ogni misura di campo magnetico si può dedurre solo $B$, a $H$ non può rimanere altro che un significato formale di relazione fra intensità di corrente e distanze, per cui anche a $\chi$ andrebbe a rigore attribuito un significato puramente formale. Per ovviare a questo inconveniente occorrerà in futuro impostare lo studio delle proprietà magnetiche della materia su di una relazione fra $I$ e $B$, come del resto $\dot{e}$ in parte già stato fatto da vari Autori, ad es. Stratton. Nel presente lavoro non si potrà fare altro che usare il vecchio concetto di suscettività, senza tuttavia alcun pregiudizio per la comprensione; inoltre, essendo in questa sede sufficiente la conoscenza dell'ordine di grandezza di $\chi$, il fattore $4 \pi$ di trasformazione trà unità u.e.m. e unità S.I. sarà assunto uguale a 10. 


\section{Analisi del ampo geomagnetico}

\subsection{DIPOLO MAGNETICO.}

Richiamiamo brevemente alcune nozioni sul dipolo magnetico, che ci saranno utili nel seguito.

Assumiamo un sistema di coordinate polari in un piano, $r, \vartheta$, il cui asse polare coincida, in direzione e verso, col momento $\boldsymbol{M}$ del dipolo e la cui origine coincida col centro di questo. E facile dimostrare che il potenziale magnetico prorlotto dal dipolo in un punto $\mathbf{P}$ di coordinate $r, \vartheta$ (fig. 1.1) ̀̀

$$
\mathrm{V}=\frac{\boldsymbol{M} \times \boldsymbol{r}}{r^{3}}=\frac{M \cos \vartheta}{r^{2}}
$$

Per ragioni di simmetria ciò vale per qualunque piano contenente l'asse polare.

In ognuno di questi piani si possono definire due particolari componenti del campo magnetico: la componente trasversale $H$, normale a $r$ e diretta nel verso di $\vartheta$ crescente (che in geomagnetismo corrisponde alla componente orizzontale), e la componente radiale $Z$, diretta nel verso assunto come positivo per $r$ (che in geomagnetismo corrisponde alla componente

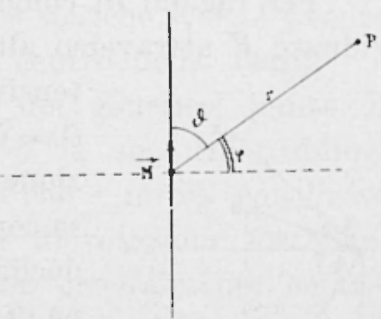

Fig. 1.1 verticale). Per le definizioni di potenziale e di campo, la prima si ottiene eseguendo la derivata direzionale $-\partial V / r \partial \vartheta$ lungo l'elemento di linea $r \partial \vartheta$ normale a $r$, Ia seconda dalla derivata $-\partial V / \partial r$. Si ha quindi

$$
\begin{gathered}
H=\frac{M \sin \vartheta}{r^{3}} \quad ; \quad Z=\frac{2 M \cos \vartheta}{r^{3}} \text { (u.e.m.) } \\
H=\frac{\mu_{0}}{4 \pi} \frac{M \sin \vartheta}{r^{3}} \quad ; \quad Z=\frac{\mu_{o}}{4 \pi} \frac{2 M \cos \vartheta}{r^{3}} \text { (S }
\end{gathered}
$$


Introducendo, in luogo della colatitudine $\vartheta$, la latitudine $\varphi$, si ha

$$
\begin{gathered}
H=\frac{M \cos \varphi}{r^{3}} ; Z=\frac{2 . U \sin \varphi}{r^{3}} \text { (u.e.m.) } \\
H=\frac{\mu_{0}}{4 \pi} \frac{M \cos \varphi}{r^{3}} ; Z=\frac{\mu_{0}}{4 \pi} \frac{2 M \sin \varphi}{r^{3}} \text { (S.I.) }
\end{gathered}
$$

\subsection{FLEMENTI DEL, CAMPO ('EOMAGNETICO.}

Il campo magnetico terrestre, o campo geomagnetico, ì un campo vettoriale, caratterizzato da un vettore, funzione del punto, che nel seguito verrà indicato con $\boldsymbol{F}$. Per individuare completamente $\boldsymbol{F}$ in un determinato punto è necessario determinarne tre componenti rispetto all un opportuno sistema di riferimento; se il punto giace sulla superficie terrestre, il sistema di riferimento può essere convenientemente scelto con l'origine nel punto stesso, l'asse $x$ nella direzione del nord geografico, l'asse y nella direzione dell'est geografico, l'asse $z$ nella direzione della verticale verso il basso. Ise componenti lungo i tre assi vengono allora indicate rispettivamente con $X, Y, Z$.

Per ragioni di comodità di misurazione si preferisce spesso indivilluare $F$ attraverso altri elementi, e precisamente un elemento in-

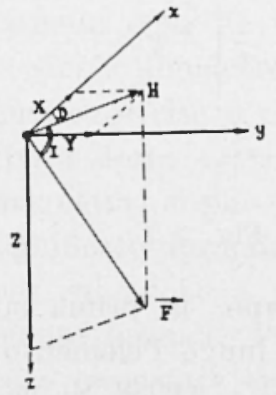

Fig. 1.2 tensivo e due elementi angolari. L'elemento intensivo è la componente orizzontale $\boldsymbol{H}$, cioc̀ la proiezione di $\boldsymbol{F}$ sul piano orizzontale, considerata anch'essa come un vettore; gli elementi angolari sono: 1) la declinazione $D$, definita come l'angolo tra la direzione di $H$ e la direzione del nord geografico, assunto come positivo quando $H$ ì diretta a est del meridliano geografico; se indichiamo col nome di meridiano magnetico di un punto il piano verticale passante per il punto stesso e contenente $F$ (e quindi $\boldsymbol{H})$, la declinazione si può definire come l'angolo fra il meridiano magnetico e il meridiano geografico; 2) la inclinazione $I$, definita come l'angolo che $\boldsymbol{F}$ forma col piano orizzontale o, ciò che è lo stesso, come l'angolo fra la direzione di $\boldsymbol{F}$ e la direzione di $\boldsymbol{H}$. La fig. 1.2 illustra gli elementi sopra definiti. 
Je relazioni fra i vari elementi sono le seguenti:

$$
\begin{gathered}
H=F^{\top} \cos I ; Z=F^{1} \sin I ; Z=H \operatorname{tg} I ; Y=H \cos I ; Y=H \sin l \\
H=\left(X^{2}+Y^{2}\right)^{1 / 2} ; F=\left(X^{2}+Y^{2}+Z^{2}\right)^{1 / 2}=\left(H^{2}+Z^{2}\right)^{1 / 2} .
\end{gathered}
$$

1.3. ANALISI DEL CAMPO GEOMAGNET(O) IN ARMONICHE SFERICHE.

Il potenziale $V$ rovuto a no certa distribuzione di materiale magnetico (elettrico, gravitazionale) soddisfa, nei punti esterni a tale distribuzione, all'equazione di Isaplace

$$
\nabla^{2} V=0
$$

Usando un sistema di coordinate cartesiane ortogonali $x, y, z$ la $[1.2]$ si scrive

$$
\frac{\partial^{2} V}{\partial x^{2}}+\frac{\partial^{2} V}{\partial y^{2}}+\frac{\partial^{2} V}{\partial z^{2}}=0
$$

Nell'applicazione dell'equazione di Iaplace al magnetismo terrestre conviene usare un sistema di coordinate polari sferiche $r, \vartheta, \lambda$ avente

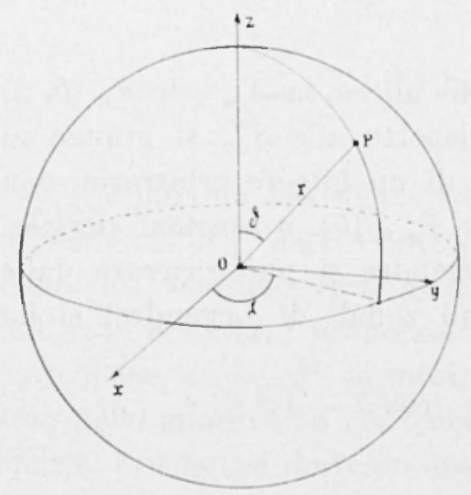

Fig. 1.3

l'origine nel centro della Terra; $r$ ì la distanza del generico punto $P$ dal centro, $\vartheta$ la sua colatitudine, cioè l'angolo che $r$ forma con l'asse polare (l'asse di rotazione terrestre, che si assume generalmente coincidente con l'asse $z$ del sistema di coorlinate cartesiane, con verso dal polo surl al polo nord) e $\lambda$ la sua longiturline, vale a dire l'angolo che il merisliano passante per $P$ forma con un meridiano di riferimento (il meridiano di Greenwich, che per convenzione si assume come contenente l'asse $x$ ) (fig. 1.3). E ovvio che il sistema di riferimento $x$, $y, z$ qui definito non ha nulla a che fare col sistema introdotto nel paragrafo precerlente. 
Le relazioni che legano le coordinate cartesiane con le coordinate polari sono le seguenti:

$$
r-r \sin \theta \cos \lambda ; \quad y=r \sin \theta \sin \lambda ; \quad z=r \cos \theta .
$$

Si dimostra che in coorlinate sferiche l'equazione di Isaplace assume la formis.

$$
\nabla^{2} V=\frac{1}{r^{2}} \frac{\partial}{\partial r}\left(,-\frac{\partial V}{\partial r}\right)+\frac{1}{r^{2} \sin \theta} \frac{\partial}{\partial \eta}\left(\sin \theta \frac{\partial V}{\partial \eta}\right)+\frac{1}{r^{2} \sin 2 \theta} \frac{\partial^{2} V}{\partial \lambda^{2}}=0
$$

Ogni funzione che soddisti allequazione di Isaplace prende il nome di funzione armonica.

Una funzione armonica omogenea di gralo $n$ in $x, y, z$ (si considerano solo valori interi positivi di $n$ ) si può scrivere, attraverso le [1.3] nella forma ${ }^{n} F_{n}(\theta, \lambda)$; la più generale soluzione dellequazione di Iaplace contiene termini di tutti i grali. Ia $r^{n} F_{n}(\vartheta, \lambda)$ viene chiamata funzione armonica sferica di gralo $n$. Si può facilmente dimostrare che, se $r^{n} F_{n}(\vartheta, \lambda)$ è una soluzione della $[1.4]$, lo è pure la $\frac{1}{r^{(n+1)}} \boldsymbol{F}_{n}(\vartheta, \lambda)$.

Sviluppando la $F_{n}(9, \lambda)$ per mezzo delle $[1.3]$, si trova che essa, contenendo $\sin \theta, \cos \theta, \sin \lambda, \cos \lambda$. lino al graulo $n$, assume la forma

$$
F_{n}=\sum_{m=0}^{n} S_{\eta}^{\prime \prime \prime} \cos \left(m \lambda+\varepsilon_{n}^{m}\right)
$$

dove $S_{n}^{\prime \prime}$ sono funzioni di 9 . Sostituendo alloma la $V_{n}=r^{n} F_{n}(\theta, \lambda)$ nella [1.4] e risolvendo tale equazione rispetto alle $s_{m}$. si giunge a] risultato che queste coincidono, a meno di un fattore arbitrario, con le cosiclette funzioni associate di Legendre $P_{n, m}(\vartheta)$, o funzioni sferiche. Ia forma esplicita delle funzioni di Isegendre si può ricavare dalle espressioni seguenti: per $m=0$ (funzioni zonali di Isegendre) si ha

$$
P_{n}(\theta)=\frac{1}{n ! 2^{n}} \frac{1^{n}}{\left(1(\cos \theta)^{n}\right.}\left(\cos ^{2} \theta-1\right)^{n} ;
$$

per $m>0$ ma sempre $\leqslant n \grave{\mathrm{e}}$

$$
\bar{L}_{n, m}(\vartheta)=\sin " \bar{y} \frac{\mathrm{d}^{m} P_{n}(\vartheta)}{\mathrm{d}(\cos \vartheta)^{m}}
$$

$n$ è il grado e $m$ l'ordine delle funzioni di Juegendre. 
Nel seguito si useranno le funzioni, dette "funzioni quasi normalizzate di Schmidt n, $P_{n}^{m}(9)$ :

$$
\left.\begin{array}{l}
P_{s}^{\prime \prime \prime}(i)=P_{n . m}(\theta) \quad \text { per } m-0 \\
P_{n}^{w_{n}}(\theta)=\left\{2 \frac{(n-m) !}{(n+m) !}\right\}^{1 / 2} P_{n, m}(\theta) \text { per } m>0
\end{array}\right\}
$$

Il fattore moltiplieativo nella secondla espressione ì stato introrlotto da Schmidt per fare in modo che le funzioni $P_{1}^{\prime \prime \prime} \cos m \lambda$ e $I_{1}^{\prime \prime \prime} \sin m \lambda$. (che compaiono nello sviluppo della [1.5] e vengono chiamate funzioni armoniche superficiali) siano dello stesso ordine di grandezza delle funzioni zonali $P_{n}(m=0)$ di uguale grado. Si ha infatti dlalle [1.ti] che sia il valore medio di $P_{n}^{2}$ su di una superficie sferica, sia il valore merlio di $\left(P_{n}^{m \prime} \cos m \lambda\right)^{2}$ o di $\left(P_{n}^{\prime \prime \prime} \sin m \lambda\right)^{2}$ sulla stessa sferal sono dati da $\frac{1}{2 n+1}$.

Dalla [1.5] si la che $F_{n}$ può essere scritta nella forma

$$
F_{n}=\sum_{m=0}^{n}\left(A_{\prime \prime \prime}^{\prime \prime} \cos m \lambda+B_{i}^{m} \sin m \lambda\right) P_{n}^{m}(\theta)
$$

dove $A_{1}^{m}$ e $B_{1}^{m}$ sono coefficienti indipendenti da $\theta$ e da $\lambda$. Il potenziale $V^{r}$ sulla superficie di una sfera di raggio unitario viene espresso dalla somma di infiniti termini $F_{n}$ per $"$ variabile da 1 a $\infty$ :

$$
V=\sum_{n=1}^{\infty} \sum_{m=n}^{n}\left(A_{n}^{m} \cos m \lambda_{t}+B_{t}^{m} \sin m \lambda_{n}\right) P_{n}^{m}(\theta)
$$

Assumiamo ora una sfera di raggio a, e consideriamo la duplice dipendenza di $V$ da $r$. Come si è dianzi rletto, la soluzione più generale della [1.2] contiene termini di tutti i gradi, quindi termini proporzionali a $r^{n}$ e termini proporzionali a $1 / r^{(n+1)}$. Per comprentere il signifirato fisico di questi termini, occorre ricordare che la [1.2] i valida solo nello spazio non contenente la materia che produce $V$; in questo spazio $\mathrm{V}$ e le sue derivate spaziali (le componenti della forza) si mantengono sempre finite. Se la materia è contenuta in tutta e sola la sfera di raggio $a$, la [1.2] è valida per $r \geqslant a$; affinché $V$ e la forza siano finite, tendendo a zero per $r \rightarrow \infty$, occorre che nello sviluppo di $r$ non compaiano potenze positive di $r$. Il potenziale deve quindi contenere solo termini della forma $(a / r)^{(n+1)}$; questi rappresentano perciò 
il potenziale di origine interna alla sfera. Se invece la materia è esterna alla sfera di raggrio $a$, la $[1.2]$ i valida per $r<a$; se $V$ deve rimanere sempre finito, la sua espressione deve contenere solo termini della forma $(r / a)^{n}$, che rappresentano percio il potenziale di origino esterma. I'espressione generale del potenziale $V$ si può scrivere allora

$$
\begin{aligned}
V= & a \sum_{n=1}^{\infty} \sum_{m=0}^{n} \mid\left\{r_{\prime \prime}^{\prime \prime}\left(\frac{r}{a}\right)^{n}+\left(1-c_{n}^{m}\right)\left(\frac{a}{r}\right)^{n+1}\right\} A_{n}^{\prime \prime \prime} \cos m \lambda+ \\
& +\left\{s_{n}^{m}\left(\frac{r}{a}\right)^{\prime \prime}+\left(1-s_{n}^{\prime \prime \prime}\right)\left(\frac{a}{r}\right)^{n+1}\right\} B_{n}^{m} \sin m \lambda_{n} P_{n}^{\prime \prime \prime}(\theta),
\end{aligned}
$$

in cui $e_{1}^{m \prime}$ e $s_{n}^{m}$, compresi fra 0 e 1 , inclicano la fraziono dei termini armonici in $\cos m \lambda$ e sin $m \lambda$. dovuta a materia esterna alla sfera; il fattore a fuori dai segni di sommatoria serve a dare ai coefficienti $A_{n}^{\prime \prime \prime} . B_{\imath}^{\prime \prime \prime}$ le dimensioni dell’intensita di un campo (magnetico, elettrico, gravitazionale).

Nelle applicazioni al geomagnetismo si è soliti porre

$c_{n}^{m} A_{n}^{m}=g_{n}^{m e}, \quad\left(1-c_{n}^{m}\right) A_{n}^{m}=g_{n}^{m i}, \quad s_{n}^{m} B_{n}^{m}=h_{n}^{m e}, \quad\left(1-s_{n}^{m}\right) B_{n}^{m}=h_{n}^{m i}$

da cui

$$
g_{n}^{m e}+g_{n}^{m i}=g_{n}^{m \prime}=A_{n}^{\prime \prime \prime} ; h_{n}^{m e}+h_{n}^{m i}=h_{n}^{m}=B_{n}^{m i},
$$

dove i suffissi e, i denotano esterno e interno rispettivamente. Allora $P$ si scrive

$$
V=a \sum_{n=1}^{\infty}\left\{\left(\frac{r}{a}\right)^{n} T_{n}^{e}+\left(\frac{a}{r}\right)^{n+1} T_{n}^{i}\right\}
$$

dove $a$ mppresenta ora il raggio terrestre, mentre la notazione $T_{n}$ (indipendentemente dai sufiissi) indica l'espressione

$$
T_{n}=\sum_{m=0}^{n}\left(g_{n}^{m} \cos m \lambda+h_{n}^{m} \sin m \lambda\right) P_{r}^{m}(\eta) .
$$

$g_{n}^{m}$ e $h_{n}^{m}$ vengono chiamati coefficienti di Gauss.

E importante sottolineare che la [1.7], che noi abbiamo dedotto a partire dall'equazione di Iaplace, cioè cla una legge fisica, può anche essere considerata, indipendentemente da tale legge, semplicemente come la rappresentazione di una funzione spaziale su di una superficie 
sferica, esattamente come uno sviluppo in serie di Fourier rappresenta una funzione di una variabile in un determinato intervallo di questa. Anche ciascuna componente del campo greomagnetico potrebbe quindi essere espressa in forma analoga alla [1.7]; ma se il campo deriva da un potenziale, caratterizzato dai coefficienti di Gauss $g_{n}^{\prime \prime}$ e $h_{n}^{m}$ sopra definiti, questi dovranno comparire anche nelle espressioni delle componenti del campo dedotte calcolando le derivate parziali, cambiate di segno, di $r(r, \vartheta, \lambda)$ rispetto alle direzioni degli assi $x, y, z$ del sistema di riferimento definito in 1.2 (non sari inutile osservare che questo sistema, se riportato in fig. 1.3 , avrebbe la sua origine in $P$, l'asse $z$ coincidente con $r$ e il piano $x y$ tangente in $P$ alla sfera, con $x$ diretto verso il polo nord). Tenendo conto del segno di $X$ (positivo verso nord), di $\vartheta$ (crescente a partire dall'asse polare) e di $Z$ (positivo verso il centro (lella Terra), si ha:

$$
\begin{aligned}
& \left.X=\frac{\partial V}{r \partial \vartheta}=\frac{a}{r} \sum_{n=1}^{\infty} \sum_{m=0}^{n} \frac{\mathrm{d} P_{n}^{m}}{\mathrm{~d} \vartheta} \mid g_{n}^{m e}\left(\frac{r}{a}\right)^{n}+g_{n}^{m i}\left(\frac{a}{r}\right)^{n+1}\right\} \cos m \lambda+ \\
& \left.+\left\{7_{n}^{m c}\left(\frac{r}{a}\right)^{n}+i_{n}^{m}\left(\frac{a}{r}\right)^{n+1}\right\} \sin m \lambda\right] \\
& Y=-\frac{\partial V}{r \sin \theta \mathrm{d} \lambda}=\frac{a}{r \sin \vartheta} \sum_{n=1}^{\infty} \sum_{m=0}^{n} P_{n}^{m}\left[m\left\{g_{n}^{m e}\left(\frac{r}{a}\right)^{\prime \prime}+g_{n}^{m i}\left(\frac{a}{r}\right)^{n+1}\right\}\right. \\
& \left.\sin m \lambda-m\left\{h_{n}^{m e}\left(\frac{\ddot{a}}{a}\right)^{n}+h_{n}^{m i}\left(\frac{a}{r}\right)^{n+1}\right\} \cos m \lambda\right\} \\
& Z=\frac{\partial V}{\partial r}=\sum_{n=1}^{\infty} \sum_{m=0}^{n} I_{n}^{m} \mid\left\{n g_{n}^{n e}\left(\frac{r}{a}\right)^{i=}-(n+1) g_{n}^{n i i}\left(\frac{a}{r}\right)^{n+2}\right\} \cos m \lambda+ \\
& +\left\{n h_{n}^{m e}\left(\frac{r}{a}\right)^{n-1}-(n+1) h_{n}^{m i}\left(\frac{a}{r}\right)^{n+2} \sin m \lambda\right] \text {. }
\end{aligned}
$$

Sulla superficie della Terra, cioè per $r=a$, le precedenti divengono, tenendo conto delle $\left[1.8^{\prime}\right]$ :

$$
\begin{aligned}
& X=\sum_{n=1}^{\infty} \sum_{m=0}^{n} \frac{1 P_{n}^{m}}{a \vartheta}\left|g_{n}^{m} \cos m \lambda+h_{n}^{n} \sin m \lambda\right| \\
& Y=\frac{i .}{\sin \vartheta} \sum_{n-1}^{\infty} \sum_{m=0}^{n} P_{n}^{m}\left[m g_{n}^{m} \sin m \lambda-m h_{\imath}^{\prime \prime} \cos m \lambda\right]
\end{aligned}
$$




$$
\begin{gathered}
Z=\sum_{n=1}^{\infty} \sum_{m=0}^{n} P_{a}^{m}\left[\left\{n g_{n}^{m e}-(n+1) g_{n}^{m}\right\} \cos m \lambda+\right. \\
\left.+\left\{n h_{n}^{m e}-(n+1) h_{n}^{m}\right\} \sin m \lambda^{\prime}\right\}
\end{gathered}
$$

Dalla distribuzione dei valori di $X$ e $Y$ sulla superficie ter'estre si possono ottenere, per le $\left[1.9^{\prime}\right]$, i coefficienti $q_{n}^{m}$ e $h_{n}^{m}$. I tale scopo il metodo più largamente usato è stato il seguente.

Dai valori misurati nelle varie stazioni, generalmente distribuite pinttosto irregolarmente sulla superficie terrestre a causa delle condizioni geografiche, si tracciano le isodinamiche per le varie componenti; per interpolazione si ottengono poi i valori di queste su di una rete regolare costituita dall'incrocio di determinati paralleli e determinati meridiani, ad esempio ogni $10^{\circ}$ di latitudine e di longitudine o più fitti (ogni $5^{0}$ di latitudine e di longitudine), a seconda clel materiale sperimentale a disposizione. Per ogni parallelo, ciò per un prefissato valore di $\vartheta$, si esegue un normale sviluppo in serie di Fourier di una componente, che indicheremo con $f$, per punti equiclistribuiti su quel parallelo; si ottengono in tal modo i coefficienti cli Fourier $a_{m}, b_{m}$ della espressione

$$
f(\vartheta)=\sum_{m=0}^{\infty}\left(a_{m} \cos m \lambda+b_{m} \sin m \lambda\right)
$$

Si uguagliano allora, per quel determinato valore di $\vartheta$, gli $a_{m}, b_{m}$ con i corrispondenti coefficienti di $\cos m \lambda$ e $\sin m \lambda$ nelle [1.9']; facendo cio per ogni valore di $\vartheta$ prescelto si ottiene un sistema di equazioni per ciascuno dei due gruppi di incognite $g_{\eta}^{m}$ e $h_{\eta}^{m}$. Illustriamo il metodo per i $g_{n}^{0}$, per es. per la $X: a_{0}$ è allora dato da

$$
a_{o}=\frac{\mathrm{d} P_{1}^{0}}{\mathrm{~d} \theta} g_{1}^{0}+\frac{\mathrm{d} P_{2}^{0}}{\mathrm{~d} \theta} g_{2}^{0}+\frac{\mathrm{d} P_{3}^{0}}{\mathrm{l} \vartheta} g_{3}^{0}+\ldots \ldots
$$

dove le $\frac{d P_{n}^{0}}{d \vartheta^{2}}$ sono coefficienti costanti noti, essendo fissato il valore di $\vartheta$. Ripetendo il procerlimento per ognuno dei paralleli scelti, si ottiene un sistema di equazioni nelle incognite $g_{n}^{0}$ che è risolubile se $n$ è minore o uguale al numero dei paralleli, cioè al numero delle equazioni; normalmente ci si limita a un gralo $n$ minore del numero delle equazioni, per cui il sistema è risolubile mediante il metorlo dei minimi quadrati. 
Allo stesso modo si ottengono i coefficienti di ordine superiore $g_{n}^{m}$ e $h_{n}^{n}$, tenendo ben presente che in questo caso nelle equazioni non possono comparire termini, nei quali sia $m>n$, per la definizione delle $P_{n}^{m}$ o rlelle $P_{n, m}$ data a pag. 14 .

Se il campo deriva rigorosamente da un potenziale, è evidente che i coefficienti $\eta_{n}^{\prime \prime}$ e $h_{1}^{\prime \prime \prime}$ dedotti dalle componenti $X$ e $Y$ separatamente devono coincidere; per valutare questa coincidenza occorre naturalmente tener conto degli errori di misura, degli errori di cartografia (tracciatura delle isodinamiche), degli errori di interpolazione ecc. Un accurato esame dei risultati di tutte le analisi finora eseguite porta a concludere che le differenze riscontrate nei coefficienti calcolati da $X$ e da $Y$ possono attribuirsi appunto solo agli errori di cui sopra. Come coefficienti $g_{1}^{\prime \prime}, h_{1}^{\prime \prime}$ si assumono le medie dei valori trovati nelle analisi di $\mathrm{I}$ e di $\mathrm{I}$, se necessario opportunamente pesati.

Al tempo delle prime analisi, quando l'imprecisione dei risultati non permetteva di escludere dal campo geomagnetico una parte non derivante da un potenziale, era stata avanzata l'ipotesi dell'esistenza. di correnti elettriche provenienti clall'esterno e attraversanti la superficie della Terra. I noto, infatti, che un campo non conservativo dà luogo a circuitazione diversa da zero e la circuitazione non nulla di un campo magnetico indica l'esistenza di una corrente elettrica concatenata col circuito lungo il quale è calcolata la circuitazione, secondo la relazione

$$
\oint B \times d l=\mu_{a} I
$$

scegliendo il percorso sulla superficie terrestre, si poteva pensare di calcolare l'intensità delle correnti aria-terra. Ma per quanto si è detto sopra, questa ipotesi è oggi praticamente caduta. Esiste tuttavia una corrente elettrica verticale che dall'atmosfera scorre verso il suolo, come sappiamo dagli studi di elettricita atmosferica. Ma tenendo conto che la densità $J$ di tale corrente è dell'ordine di $3.10^{-12} \mathrm{~A} / \mathrm{m}^{2}$, possiamo calcolare l'ordine di grandezza del campo magnetico prodotto da essa lungo il perimetro di una superficie circolare dell'area di $1 \mathrm{~km}^{2}$ $\left(r=5,6.10^{2} \mathrm{~m}\right)$ dalle relazioni

$$
\begin{gathered}
B \cdot 2 \pi r=\mu_{0} \mathrm{JS}=4 \pi \cdot 10^{-7} \mathrm{JS} \\
B=\frac{2 \cdot 10^{-7} \cdot 3 \cdot 10^{-12} \cdot 10^{6}}{r}=\frac{6 \cdot 10^{-13}}{r} \approx 10^{-15} \mathrm{~T}=10^{-6} \mathrm{nT}(\gamma) .
\end{gathered}
$$

I'intensità dlel campo magnetico ì talmente inferiore alle possibilitì di una determinazione sperimentale, che le suddette correnti verticali 
non possono in alcun modo influire sul campo magnetico terrestre da noi conosciuto.

\subsection{SEPARAZIONE DEI CAMPI DI ORIGINE INTERNA E DI ORIGINE ESTERNA.}

Come si vede, dall'analisi delle due componenti orizzontali $X$ e $Y$ misurate sulla superficie terrestre $(r=a)$ si possono dedurre solo i coefficienti $g_{n}^{m}, h_{2}^{n}$ che, per le $\left[1.8^{\prime}\right]$, sono la somma dei coefficienti interni erl esterni. Per determinare separatamente i contributi dei campi di origine interna e di origine esterna occorre analizzare anche la distribuzione della componente verticale $Z$; per le [1.8] e [1.8'] si puó scrivere per $Z$ sulla superficie terrestre

$$
\begin{gathered}
Z=\sum_{n=1}^{\infty} \sum_{m=0}^{n} P_{n}^{m}(\vartheta) \mid\left\{n c_{n}^{m}-(n+1)\left(1-c_{n}^{m}\right)\right\} g_{n}^{m} \cos m \lambda+ \\
\left.+\left\{n s_{n}^{m}-(n+1)\left(1-s_{n}^{m}\right)\right\} h_{n}^{m} \sin m \lambda\right]
\end{gathered}
$$

d'altra parte, eseguendo anche su $Z$ lo sviluppo in armoniche sferiche descritto precedentemente, si ottiene

$$
Z=\sum_{n=1}^{\infty} \sum_{m=0}^{n} P_{n}^{\prime}(\vartheta)\left(\alpha_{n}^{m} \cos m \lambda+\beta_{n}^{r^{n}} \sin m \lambda\right)
$$

dove gli $\alpha_{\imath}^{m}$ e $\beta_{n}^{\prime \prime}$, dedotti in modo analogo ai $q_{r}^{m}$ e $h_{n}^{m}$ delle componenti $X$ e $Y$, sono ora uguali a

$$
\begin{aligned}
& \alpha_{n}^{m}=\left\{n c_{n}^{m}-(n+1)\left(1-c_{n}^{m}\right)\right\} g_{n}^{m} \\
& \beta_{n}^{m}=\left\{n s_{n}^{m}-(n+1)\left(1-s_{n}^{m}\right)\right\} h_{n}^{m}
\end{aligned}
$$

In queste equazioni sono noti $\alpha_{\eta}^{n}$ e $\beta_{1}^{m}$, testé calcolati dallo sviluppo di $Z$, come pure $g_{n}^{m}$ e $h_{n}^{m}$, derlotti dallo sviluppo di $X$ e di $Y$; esse permettono quindi di calcolare $c_{n}^{m}$ e $s_{n}^{m}$, cioè la frazione dei termini armonici di origine esterna.

\subsection{Risultati DELle PRINCIPALI ANALisi.}

Dal 1830 ad oggi (cioè dal tempo in cui Gauss per primo usò lo sviluppo in armoniche sferiche per l'analisi del campo geomagnetico) 
sono state eseguite una trentina di analisi. Ventisei di esse sono state criticamente discusse da McDonald e Gunst (1967); nella Tab. 1 ne sono riportati i risultati, sotto forma di valori dei coefficienti di Gauss fino a $n=m=2$; le singole analisi, tuttavia, sono state estese a gradi e ordini ben superiori. Ad esempio, mentre l'analisi di Gauss giungeva fino a $n=m=4$, l'analisi di Hurwitz e coll. si spinge fino a $n=$ $m=12$. Le analisi si sono moltiplicate negli ultimi venti anni, allo scopo di cercare un sempre più accurato modello del campo geomagnetico da estrapolare all'esterno della Terra in vista degli studi sulla magnetosfera e sul comportamento dei plasmi e del vento solare nel campo geomagnetico stesso.

Va notato che non tutti gli Autori usano le funzioni quasi-normalizzate di Schmidt $P_{\imath}^{m}(\vartheta)$; Gauss ha usato le funzioni da lui denotate $P^{n, m}$, che con le funzioni associate di Legendre $P_{n, m}$ hanno la relazione seguente

$$
P_{n, m}=\frac{(2 n) !}{2^{n} \cdot n !(n-m) !} P^{n, m}
$$

le stesse funzioni usano Dyson e Furner, nonché Jones e Melotte. I coefficienti di Gauss della tabella sono stati tuttavia riportati tutti allo stesso fattore di normalizzazione, e sono quindi direttamente confrontabili fra loro.

Vi sarebbero però altri punti da discutere riguardo alla confrontabilità delle varie analisi. $\mathrm{E}$ certo, infatti, che dai tempi di Gauss a oggi la situazione si è nettamente modificata riguardo sia alla precisione strumentale, sia al numero e alla distribuzione delle stazioni, fattori questi che intervengono in misura notevole nella determinazione dei coefficienti di Gauss; inoltre le analisi, anche le più recenti, si avvalgono di dati originali alle volte diversi, alle volte uguali ma pesati e analizzati con criteri e procedimenti diversi. Un altro elemento importante è risultato il grado e l'ordine massimi presi in esame: sembra infatti accertato che, mentre per $n, m$ massimi $\geqslant 6$ i coefficienti ottenuti trattando determinati dati con un determinato procedimento sono praticamente indipendenti dai valori massimi di $n$ e $m$ scelti, il troncare la serie di armoniche sferiche a valori minori di 6 influisce sui valori calcolati dei coefficienti.

Un esempio delle differenze che le varie cause ora accennate possono produrre è dato dall'analisi di Gauss confrontata con una sua rielaborazione morlerna. La prima analisi di Gauss, ridotta, se- 
Tab. 1 - Coefficienti di Gauss di dipolo e quAdrupolo (in gauss $=10^{-4} T^{\prime}$ ), monento di dipolo, aNgoli polalke E azimutale dell'asse DEL Dipolo (da MeDonald e Gunst, 1967).

\begin{tabular}{|c|c|c|c|c|c|c|c|c|c|c|c|c|}
\hline Autori & Epoca & $g_{1}^{0}$ & $\mathfrak{r}_{1}^{1}$ & $l_{1}{ }_{1}^{1}$ & $\underline{g}_{2}^{0}$ & $\mathrm{~g}_{2}^{1}$ & $\mathrm{~g}_{\mathrm{z}}^{-}$ & $h_{2}^{1}$ & $\mathrm{l}_{2}^{2}$ & $\begin{array}{c}\mathrm{M} \\
\times 10^{25} \mathrm{uem} \\
\mathrm{x} 10^{22} \mathrm{~S} . \mathrm{I} .\end{array}$ & $\begin{array}{c}9 \\
\text { polare }\end{array}$ & $\begin{array}{c}9 \\
\text { azimut }\end{array}$ \\
\hline $\begin{array}{l}\text { Erman-Petersen } \\
\text { Gauss } \\
\text { Adams } \\
\text { Adams } \\
\text { Neumayer } \\
\text { Fritsche } \\
\text { Schmidt }:\end{array}$ & $\begin{array}{l}1829 \\
1835 \\
1845 \\
1880 \\
1880 \\
1885 \\
1885 \\
1922 \\
1942.5 \\
1905 \\
1915 \\
1925 \\
1935 \\
1945 \\
1945 \\
1945 \\
1945 \\
1955 \\
1955 \\
1958.5 \\
1959 \\
1960 \\
1960 \\
1960 \\
1965 \\
1965\end{array}$ & $\begin{array}{l}-.3201 \\
-.3235 \\
-.3219 \\
-.3168 \\
-.3157 \\
-.3164 \\
-.3174 \\
-.3095 \\
-.3039 \\
-.31423 \\
-.31176 \\
-.30892 \\
-.30662 \\
-.30570 \\
-.3032 \\
-.3057 \\
-.30668 \\
-.3046 \\
-.3055 \\
-.3045 \\
-.30674 \\
-.30509 \\
-.3046 \\
-.3041 \\
-.30375 \\
-.30388\end{array}$ & $\begin{array}{l}-.0284 \\
-.0311 \\
-.0278 \\
-.0243 \\
-.0248 \\
-.0241 \\
-.0236 \\
-.0226 \\
-.0218 \\
-.02270 \\
-.02176 \\
-.02166 \\
-.02129 \\
-.02116 \\
-.0229 \\
-.0219 \\
-.02160 \\
-.0212 \\
-.0227 \\
-.0222 \\
-.01923 \\
-.02181 \\
-.0214 \\
-.02147 \\
-.02087 \\
-.02117\end{array}$ & $\begin{array}{l}.0601 \\
.0625 \\
.0578 \\
.0603 \\
.0603 \\
.0591 \\
.0598 \\
.0592 \\
.0555 \\
.05981 \\
.05912 \\
.05839 \\
.05792 \\
.05805 \\
.0590 \\
.0579 \\
.0577 \\
.0576 \\
.0590 \\
.0584 \\
.05762 \\
.05841 \\
.0580 \\
.05799 \\
.05769 \\
.05760\end{array}$ & $\begin{array}{r}-.0008 \\
.0051 \\
.0009 \\
-.0049 \\
-.0053 \\
-.0035 \\
-.0050 \\
-.00887 \\
-.0117 \\
-.00773 \\
-.00842 \\
-.00946 \\
-.01086 \\
-.01265 \\
-.0125\end{array}$ & $\begin{array}{l}.0257 \\
.0292 \\
.0284 \\
.0297 \\
.0288 \\
.0286 \\
.0278 \\
.02991 \\
.02940 \\
.02952 \\
.02940 \\
.02946 \\
.02959 \\
.02960 \\
.0288 \\
\\
.029596 \\
.0303 \\
.0295 \\
.0344 \\
.02971 \\
.0299 \\
.02959 \\
.02954 \\
.02983\end{array}$ & $\begin{array}{c}-.0014 \\
-.0002 \\
.0004 \\
-.0006 \\
.0065 \\
.0068 \\
.0065 \\
.01443 \\
.0156 \\
.01107 \\
.01345 \\
.01510 \\
.01608 \\
.01632 \\
.0150 \\
\\
.01547 \\
\\
.0158 \\
.0149 \\
.0124 \\
.01673 \\
.0164 \\
.01545 \\
.01579 \\
.01583\end{array}$ & \begin{tabular}{r}
-.0004 \\
.0012 \\
-.0010 \\
-.0075 \\
-.0075 \\
-.0075 \\
-.0071 \\
-.01241 \\
-.0150 \\
-.01051 \\
-.01144 \\
\hdashline .01284 \\
-.01460 \\
-.01658 \\
-.0146 \\
\\
-.01673 \\
-.0190 \\
-.0194 \\
-.01977 \\
-.01988 \\
-.0194 \\
-.01912 \\
-.01995 \\
-.02004
\end{tabular} & $\begin{array}{l}.0146 \\
.0157 \\
.0135 \\
.0149 \\
.0146 \\
.0142 \\
.0149 \\
.00843 \\
.0051 \\
.01156 \\
.00986 \\
.00814 \\
.00676 \\
.00535 \\
.0048\end{array}$ & $\begin{array}{l}8.454 \\
8.558 \\
8.488 \\
8.363 \\
8.336 \\
8.347 \\
8.375 \\
8.165 \\
8.009 \\
8.291 \\
8.225 \\
8.149 \\
8.088 \\
8.065 \\
8.010 \\
8.066 \\
8.090 \\
8.035 \\
8.067 \\
8.038 \\
8.086 \\
8.053 \\
8.037 \\
8.025 \\
8.013 \\
8.017\end{array}$ & $\begin{array}{l}11.70 \\
11.2 \\
11.3 \\
11.6 \\
11.7 \\
11.4 \\
11.5 \\
11.4 \\
11.1 \\
11.4 \\
11.4 \\
11.4 \\
11.4 \\
11.4 \\
11.8 \\
11.4 \\
11.4 \\
11.4 \\
11.7 \\
11.6 \\
11.2 \\
11.6 \\
11.5 \\
11.5 \\
11.4 \\
11.4 \\
11.49\end{array}$ & $\begin{array}{r}-64.7 \\
-63.5 \\
-64.3 \\
-68.1 \\
-67.6 \\
-67.8 \\
-68.5 \\
-68.8 \\
-68.5 \\
-70.0 \\
-69.9 \\
-70.0 \\
-69.9 \\
-70.0 \\
-68.8 \\
-69.3 \\
-69.5 \\
-69.8 \\
-69.0 \\
-69.2 \\
-71.5 \\
-69.5 \\
-69.7 \\
-69.7 \\
-70.1 \\
-69.8\end{array}$ \\
\hline
\end{tabular}


condo la Tab. 1, al 1835, era basata su 84 punti di misurazione e giungeva fino a $n=m=f$; il valore di $g_{1}^{0}$ risulta, come si velle dalla tabella, pari a $-0,3235$ (più esattamente a $-0,32348)$ gauss $(-3,2348$. $10^{4} \mathrm{nT}$ ). Un'analisi eseguita recentemente con i dati dell'epoca di Gauss, ma su 143 stazioni, ha condotto a un valore di $g_{1}^{0}$ di $-0,32484$ gauss $\left(-3,2484.10^{4} \mathrm{nT}\right)$; quest'ultima analisi, spinta fino a $n=m=6$, ha fornito per $y_{1}^{0}$ il valore di $-0,32056$ gauss $\left(-3,2056.10^{4} \mathrm{nT}\right)$.

Queste considerazioni permettono di rendersi conto dell'entità degli errori di cui possono essere affetti $i$ risultati delle varie analisi. La valutazione critica di questi errori porta tuttavia a concludere che nessuna delle ventisei analisi citate nella tabella è falsata in misura sostanziale, tale da infirmare la validità di quanto sarà ora discusso.

Un risultato di grande rilievo è che in tutte le analisi il termine $g_{1}^{0}$ e largamente preponderante sugli altri termini; i due termini che seguono per importanza, e ciò $h_{1}^{1}$ e $g_{1}^{1}$, sono dell'ordine rispettivamente del $20 \%$ e del $7 \%$ di $g_{1}^{0}$; gli altri termini (arl eccezione di $g_{2}^{1}$ ) sono tutti nettamente inferiori. I termini del primo graulo prevalgono quindi nettamente su quelli dei grarli e ordini superiori; vedremo pin avanti il significato di ciò.

Un altro risultato di fondamentale importanza è che, in tutte le analisi in cui è stato eseguito un calcolo separato di $g_{n}^{m n}, g_{n}^{m e}, h_{n}^{m e}$ $h_{a}^{m e}$, i termini di origine esterna (col suffisso e) sono praticamente trascurabili rispetto a quelli di origine interna. Limitandoci alle analisi più recenti, risulta (in gauss $=10^{-4} \mathrm{~T}$ ):

$$
\begin{array}{lll}
\text { Nagata e Oguti } & g_{1}^{01}=-3,045 \cdot 10^{-1} & g_{1}^{0 \mathrm{e}}=-5 \cdot 10^{-4} \\
& g_{1}^{11}=-2,22 \cdot 10^{-2} & g_{1}^{1 \mathrm{e}}=-1 \cdot 10^{-4} \\
& h_{1}^{11}=+5,84 \cdot 10^{-2} & 7_{1}^{7_{1}^{1 \mathrm{e}}}=+1 \cdot 10^{-4} \\
\text { Arlam e coll. } & g_{1}^{01}=-3,046 \cdot 10^{-1} & g_{1}^{0 \mathrm{e}}=-8 \cdot 10^{-4} \\
& g_{1}^{11}=-2,18 \cdot 10^{-2} & g_{1}^{1 \mathrm{e}}=+4 \cdot 10^{-4} \\
& h_{1}^{11}=+5,78 \cdot 10^{-2} & h_{1}^{1 \mathrm{e}}=+2 \cdot 10^{-4} \\
\text { Hurwitz e coll. } & g_{1}^{01}=-3,0368 \cdot 10^{-1} & g_{1}^{0 \mathrm{e}}=-8,2 \cdot 10^{-4} \\
& g_{1}^{11}=-2,111 \cdot 10^{-2} & g_{1}^{1 \mathrm{e}}=-2,4 \cdot 10^{-4} \\
& h_{1}^{11}=+5,755 \cdot 10^{-2} & h_{1}^{1 \mathrm{e}}=+1,7 \cdot 10^{-4}
\end{array}
$$

In sostanza nel coefficiente $\eta_{i}^{0}$ i termini di origine esterna sono dell'ordine del 2 per mille, mentre nei termini $g_{1}^{1}$ e $h_{1}^{1}$ sono al massimo 
dell'ordine del $2 \%$. Riprendendo le brevi considerazioni svolte a pag. 19 sull'influenza degli errori, si può ragionevolmente supporre che i termini di origine esterna risultino diversi da zero solo per le imperfezioni nelle analisi: le inesattezze nel tracciare le carte delle isodinamiche possono portare infatti a errori di qualche unità per cento, e inoltre le carte di $Z$, necessarie per la separazione dei campi di origine interna ed esterna sono in generale meno esatte di quella di $X$ e $Y$ (o $H$ e $D$ ) a causa della minore precisione delle singole misure. Pertanto, con lo stesso criterio con cui si è stabilito che il campo deriva da un potenziale (che cioè i coefficienti $q_{n}^{m}, h_{\imath}^{m}$ dedotti da $X$ e $Y$ presentano differenze attribuibili solo agli errori sperimentali), si può concludere che il campo geomagnetico è di origine interna alla Terra (si noti che i dati sperimentali su cui si basano le analisi sono praticamente depurati, mediante vari procedimenti, dalle variazioni rapide del campo, cioè la variazione diurna e le perturbazioni magnetiche). L'espressione del potenziale si può allora scrivere più semplicemente

$$
V=a \sum_{n=1}^{\infty} \sum_{m=0}^{n}\left(\frac{a}{r}\right)^{n+1} P_{n}^{m}(\vartheta)\left(g_{n}^{r} \cos m \lambda+h_{n}^{m} \sin m \lambda\right),
$$

nella quale i $g_{r}^{m}$ e $h_{r}^{m}$ si considerano coincidenti con i $g_{r}^{m \prime}$ e $h_{n}^{m}$.

\subsection{TERMINI DEL PRIMO GRADO: IL DIPOLO GEOM.AGNETICO.}

Consideriamo ora il significato dei termini del primo grado $(n=1)$. In questo caso si scrive

$$
V_{1}=\frac{\bar{c}^{2}}{i^{2}}\left(g_{1}^{0} P_{1}^{0}+g_{1}^{1} P_{1}^{1} \cos \lambda+h_{1}^{1} P_{1}^{1} \sin \lambda\right)
$$

Dalle espressioni (1.6) delle funzioni quasi normalizzate di Schmidt si ha:

per cui

$$
P_{1}^{0}=\cos \vartheta \quad ; \quad P_{1}^{1}-\sin \vartheta
$$

$$
V_{1}=\frac{\vec{\omega}^{0}}{i^{2}}\left(g^{0} \cos \vartheta+g_{1}^{1} \sin \vartheta \cos \lambda+h_{1}^{1} \sin \vartheta \sin \lambda\right)
$$

Poniamo ora le seguenti uguaglianze:

$$
g_{1}^{0^{2}}+g_{1}^{2}+h_{1}^{h^{2}}=H_{0}^{2} ; g_{1}^{0}=H_{0} \cos \bar{v}_{o} ; \frac{h_{1}^{1}}{g_{1}}=\operatorname{tg} \lambda_{0}=\frac{k \sin \lambda_{n}}{k \cos \lambda_{0}}
$$


con $k$ costante, che assumeremo positiva; essa si ottiene trasformando la prima delle [1.12]:

$$
g_{1}^{0^{-}}+k^{2} \cos ^{2} \lambda_{0}+k^{2} \sin ^{2} \lambda_{0}=H_{0}^{2},
$$

cioe

$$
\begin{gathered}
g_{1}^{c^{2}}+k^{2}=H_{0}^{2} \\
k^{2}=H_{o}^{2}-H_{o}^{2} \cos ^{2} \vartheta_{o} \quad(\text { per la seconda delle [1.12]) } \\
k^{2}=H_{o}^{2}\left(1-\cos ^{2} \vartheta_{o}\right)=H_{o}^{2} \sin ^{2} \vartheta_{0} \quad ; \quad k=H_{o} \sin \vartheta_{o}
\end{gathered}
$$

Abbiamo allora

$$
\begin{aligned}
& g_{1}^{1}=H_{o} \sin \theta_{o} \cos \lambda_{o} \\
& h_{1}^{1}=H_{o} \sin \theta_{o} \sin \lambda_{o}
\end{aligned}
$$

per cui otteniamo per $V_{1}$ l'espressione seguente:

$$
\begin{gathered}
V_{1}=\frac{a^{3}}{r^{2}}\left[H _ { o } \left(\cos \theta_{0} \cos \vartheta+\sin \vartheta_{o} \sin \vartheta \cos \lambda_{o} \cos \lambda+\right.\right. \\
\left.\left.+\sin \vartheta_{o} \sin \vartheta \sin \lambda_{n} \sin \lambda\right)\right]= \\
\left.=\frac{a^{3}}{r^{2}} \mid H_{o}\left\{\cos \vartheta_{o} \cos \vartheta+\sin \vartheta_{o} \sin \vartheta\left(\cos \lambda_{0} \cos \lambda+\sin \lambda_{o} \sin \lambda\right)\right\}\right]= \\
=\frac{a^{3}}{r^{2}}\left\{H_{o}\left\{\cos \vartheta_{o} \cos \vartheta+\sin \vartheta_{0} \sin \vartheta \cos \left(\lambda-\lambda_{0}\right)\right\} \mid .\right.
\end{gathered}
$$

I valori di $\vartheta_{o}$ e $\lambda_{o}$ si determinano dalle [1.12], una volta ottenuti, mediante l'analisi, i coefficienti $q_{1}^{0}, g_{1}^{1}, h_{1}^{1}$.

La [1.13] si può scrivere

$$
\mathrm{i}_{1}=\frac{a^{3} H_{o}}{r^{2}} \cos \Theta=\frac{M}{r^{2}} \cos \theta
$$

della quale, attraverso la fig. 1.4, possiamo verlere subito il significato. La [1.14] è cioè l'espressione del potenziale di un dipolo magnetico posto al centro della Terra, il cui asse interseca, dalla parte del suo polo positivo o nord, la superficie terrestre nel punto di colatitudine $\vartheta_{o}$ e longitudine $\lambda_{o}$, in modo tale che la colatitudine del punto in esame $(\vartheta, \lambda)$ rispetto all'asse del dipolo sia data da $\Theta . H_{o}$ rappresenta la com- 
ponente orizzontale del campo del dipolo sulla superficie terrestre nel piano equatoriale del dipolo stesso. Il punto di coordinate $\vartheta_{o}, \lambda_{o}$ è uno dei due poli geomagnetici. C'hiameremo d'ora in poi polo geoma-

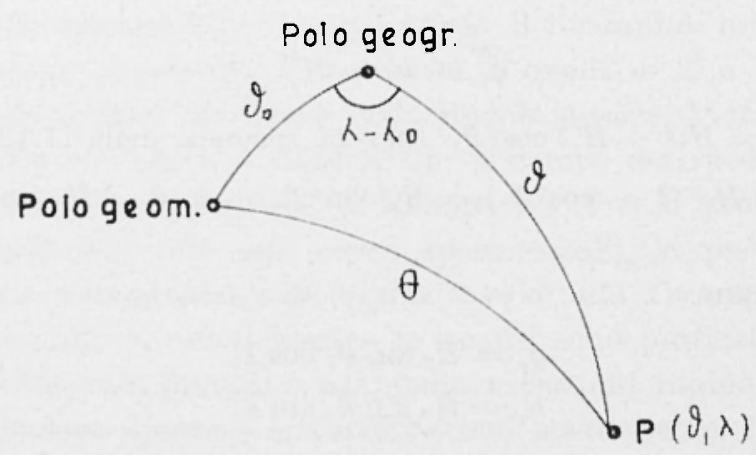

Fig. 1.4

gnetico nord quello che si trova nell'emisfero geografico nord, polo geomagnetico sud quello situato nell'emisfero sud. E importante notare che il termine $g_{1} \dot{\mathrm{e}}$, in tutte le analisi riportate, negativo; negativo è quindi anche $\cos \vartheta_{\circ}$, per cui risulta polo fisicamente positivo il polo geomagnetico sud. La sua ubicazione si deduce dal valore e dal segno dei coefficienti $g_{1}^{0}, g_{1}^{1}, h_{1}^{1}$ mediante le [1.12]; dall'analisi di Hurwitz e coll., una delle più recenti, si ha $\vartheta_{0}=168^{\circ} 35^{\prime}$ e $\lambda_{o}=110^{\circ} 11^{\prime}$. Siccome nelle analisi si assume come polo di riferimento delle colatitudini il polo geogratico nord e come meridiano di riferimento il meridiano $0^{\circ}$, o di Greenwich, le coordinate geografiche del polo geomagnetico sud sono: $\varphi=168^{\circ} 35^{\prime}-90^{\circ}=78^{\circ} 35^{\prime} \mathrm{S}, \lambda=110^{\circ} 11^{\prime} \mathrm{E}$. Il polo geomagnetico nord, che normalmente si assume come polo geomagnetico di riferimento, è diametralmente opposto al primo, per cui le sue coordinate sono: $\varphi_{0}=78^{\circ} 35^{\prime} \mathrm{N}, \lambda_{o}=290^{\circ} 11^{\prime} \mathrm{E}=69^{\circ} 49^{\prime} \mathrm{W}$. L'asse del dipolo terrestre è quindi inclinato di $110^{\circ} 5^{\prime}$ rispetto all'asse di rotazione, mentre il momento del dipolo è diretto da nord verso sud; il suo valore, che si derluce dalla prima delle [1.12] e dalla [1.14], è $8,017 \cdot 10^{25}$ u.e.m. $\left(8,017 \cdot 10^{22} \mathrm{Am}^{2}\right)$. Nella Tab. 1 sono rati i valori del momento $M$ derlotti in tutte le analisi riportate.

Un'altra rappresentazione della [1.11] si ottiene considerandone separatamente $\mathrm{i}$ tre termini. Il primo, $\frac{g_{1}^{0} a^{3}}{;} \cos \vartheta$, $\dot{a}$ il potenziale nel 
punto $P(r, \vartheta, \lambda)$ di un dipolo di momento $g_{1}^{0} a^{3}$ parallelo all'asse polare, cioè all'asse di rotazione terrestre; quanto al seconclo termine, $\frac{g_{:}^{1} a^{3}}{r^{2}} \sin \vartheta \cos \lambda$, dalla fig. 1.3 si verle che esso si può anche scrivere $\frac{g_{1}^{1} a^{3}}{r^{2}} \cos r x$, ed è quindi il potenziale nel punto $P$ di un dipolo di momento $g_{1}^{1} a^{3}$ avente come asse l'asse $x$; il terzo termine, $\frac{7_{1}^{1} n^{3}}{i^{3}} \sin \theta$

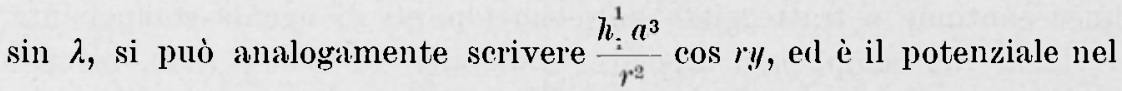
punto $P$ di un dipolo di momento $h_{1}^{1} a^{3}$ avente come asse l'asse $y$. In conclusione la [1.11] si può interpretare come la combinazione di tre dipoli ortogonali posti nel centro della Terra, uno secondo l'asse di rotazione e di momento nettamente superiore agli altri, il secondo nel piano equatoriale e diretto verso il punto $\lambda=0$, il terzo nel piano equatoriale e diretto verso il punto $\lambda=90^{\circ}$.

\subsection{I 'IERMINI DI GRADO SLPERIORE.}

Nella Tab. 1 sono riportati per ogni analisi (escluse (1ue) anche i cinque termini di grado $n=2\left(g_{2}^{0}, g_{2}^{1}, g_{2}^{2}, h_{2}^{1}, h_{2}^{2}\right)$ che sono chiamati termini di quadrupolo; ma, come si è precedentemente detto, in ogni analisi sono stati calcolati i termini di ordine superiore anche a quelli di quadrupolo, fino ad una coppia $n, m$ variabile in generale da analisi ad analisi. I termini superiori a quelli di dipolo sono talvolta inclicati col nome comprensivo di campo irregolare, lasciando il nome di campo principale al campo di dipolo.

Per il termine $n=2$ si scrive

$$
V_{2}-\frac{a^{4}}{r^{3}}\left[g_{2}^{0} P_{2}^{0}+\left(g_{2}^{1} \cos \lambda+h_{2}^{1} \sin \lambda\right) P_{2}^{1}+\left(g_{2}^{2} \cos 2 \lambda+h_{2}^{2} \sin 2 \lambda\right) P_{2}^{2}\right] \text {. }
$$

Kelvin ha dimostrato che la somma dei termini con $n=1$ e $n=2$ corrisponde a un dipolo parallelo al dipolo centrale ma spostato rispetto ad esso, più un termine in $P_{2}^{2}$. Dal valore dei coefficienti calcolati nelle analisi più aggiornate si deduce che il dipolo eccentrico c spostato rispetto al dipolo centrale di alcune centinaia di chilometri verso un punto della superficie situato in Indonesia. Questo dipolo 
eccentrico costituisce una migliore rappresentazione globale del campo visto dall'esterno della Terra; tale rappresentazione, tuttavia, non è più valida a distanze dal centro superiori a 5 o 6 raggi terrestri, dato che, come è ora noto, il campo magnetico più esterno è deformato dal vento solare, presentando uno schiacciamento dalla parte esposta al Sole e una lunga coda, di cui non si è ancora individuato il termine, dalla parte opposta.

Ina mappa del campo irregolare è riportata in fig. 1.5, dove le linee continue o tratteggiate uniscono i punti di uguale componente verticale del campo non dipolare, a distanze di $2000 \gamma$ (nT), mentre le frece indicano la componente orizzontale di detto campo in scala tale che una lunghezza pari alla distanza tra i meridiani intervallati di $10^{\circ}$ corrisponde a $6800 \gamma(\mathrm{nT})$. Tale mappa riporta in sostanza le cosidette "anomalie" del campo magnetico terrestre per le componenti $Z$ e $H$, vale a dire le differenze fra i valori delle componenti attualmente misurati e quelli che si avrebbero nei singoli punti se esistesse il solo campo geomagnetico di dipolo (centrale).

Dalla mappa si verle che le linee isoanomale formano degli ovali abbastanza regolari, comprendenti vaste regioni del globo; ma da essa non appare alcuna chiara relazione con le caratteristiche geografiche (e possiamo aggiungere anche geologiche) delle aree interessate. Ise più importanti di tali anomalie sono: l'anomalia dell'Atlantico meridionale, il eni massimo è di circa $+17000 \gamma(\mathrm{nT})$ in $Z$ e comporta una anomalia in $H$ nel Sud Afriea di circa $-12000 \gamma$ (nT); l'anomalia asiatica, con centro nella Cina dove $\Delta Z=+16000 \gamma(\mathrm{nT})$ e $\Delta H$ a nord e a sud del centro vale rispettivamente $-9000 \gamma(\mathrm{nT})$ e $+9000 \gamma$ (nT); l'anomalia del golfo di Guinea, dove $\Delta Z=-16000 \gamma(\mathrm{nT})$.

\subsection{COORINATE GEOMAGNETICHE.}

Per dedurre l'anomalia magnetica di un punto in cui si conoscano gli elementi magnetici (ad es. $H, D, Z$ ) è necessario clunque calcolare il campo geomagnetico di dipolo in quel punto. A tale scopo (e anche per motivi di carattere più generale) è utile introdurre qui la nozione di coordinate geomagnetiche.

La latitudine geomagnetica $\Phi$ è definita allo stesso modo della latitudine geografica, assumendo però come riferimento l'asse del dipolo anziché l'asse di rotazione terrestre. Sono quindi individuati i 


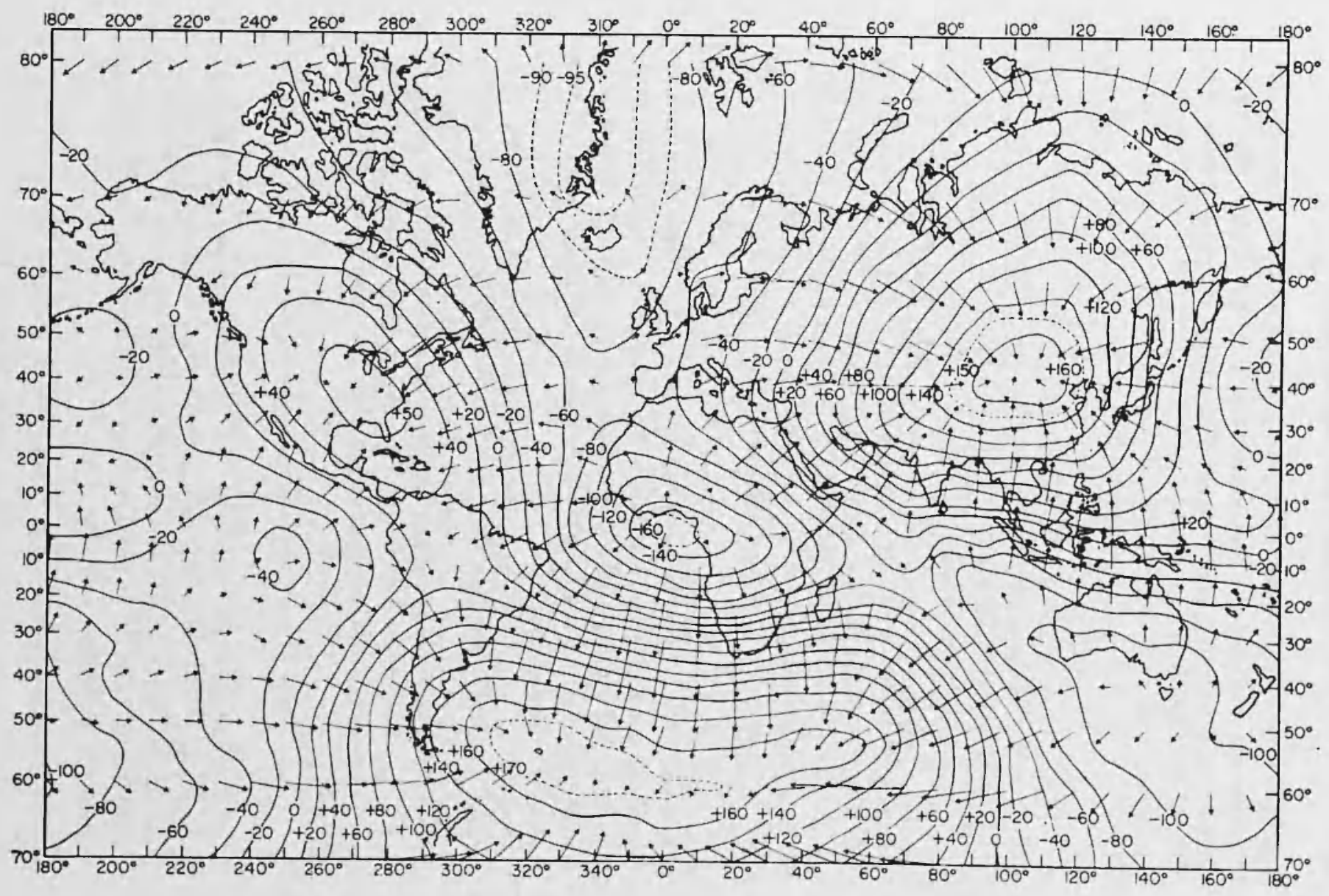

Fig. 1.5 - Campo non dipolare all'epoca 1945.0 (da Bullard e coll. 1950). 
poli geomagnetici (come si è già visto) e l'equatore geomagnetico, ciò̀ il piano perpendicolare all'asse geomagnetico nel centro del dipolo (centro della Terra). I poli geomagnetici hanno quindi latitudine geo-

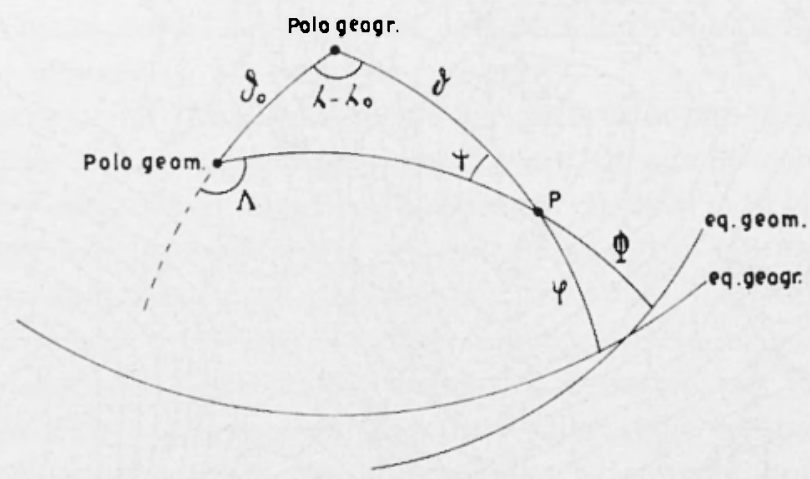

Fig. 1.6

magnetica $\Phi= \pm 90^{\circ}$ (o colatitudine $\Theta=0^{\circ}$ e $\left.180^{\circ}\right)$. Ovviamente l'equatore geomagnetico ì il luogo dei punti per i quali $\Phi=0$ (o $\Theta=90^{\circ}$ ). Dalla fig. 1.6, che ì il completamento (lella fig. 1.4, si verle che per calcolare la colatitudine geomagnetica $\Theta$ di un punto $P$ vale la seguente espressione:

$$
\cos \theta=\cos \theta \cos y_{0}+\sin \theta \sin \theta \cos \left(\lambda-\lambda_{n}\right)
$$

dove $\vartheta$ e $\lambda$ sono la colatiturline e la longiturline geografiche del punto $P$, $\vartheta_{o}$ e $\lambda_{o}$ la colatitudine e la longitudine geografiche del polo geomagnetico. Introducendo le rispettive latitudini in luogo delle colatitudini si ha

$$
\sin \Phi=\sin \varphi \sin \varphi_{o}+\cos \varphi \cos \varphi_{o} \cos \left(\lambda_{0}-\lambda_{o}\right)
$$

La longiturline geomagnetica $\Lambda$ ̀̀ l'angolo formato (verli fig. 1.6) dal meridiano geografico del polo geomagnetico e dal cerchio massimo passante per detto polo e il punto $P$, contato verso est a partire dal merirliano del polo geomagnetico. Essa si ottiene attraverso le seguenti espressioni, deducibili dalla fig. 1.6:

$$
\begin{aligned}
\cos \vartheta= & \cos \theta \cos \vartheta_{0}+\sin \theta \sin \vartheta_{0} \cos (\pi-\Lambda)= \\
& =\cos \Theta \cos \vartheta_{o}-\sin \theta \sin \vartheta_{o} \cos \Lambda
\end{aligned}
$$




$$
\cos A=\frac{\cos \theta \cos \theta_{o}-\cos \theta}{\sin \theta \sin \vartheta_{o}}
$$

Per accertare il valore di $A$ occorre, una volta calcolata la [1.16], cercare il qualrante in cui essa carle. Facendo sempre riferimento alla fig. 1.6, si deducono le relazioni

$$
\begin{aligned}
& \frac{\sin (\pi-\lambda)}{\sin \vartheta}-\frac{\sin \left|\lambda-\lambda_{n}\right|}{\sin \Theta} \\
& \sin A=\sin \left|\lambda-\lambda_{0}\right| \frac{\sin \vartheta}{\sin \theta}
\end{aligned}
$$

dove i valori $\sin \vartheta$ e $\sin \theta$ sono sempre positivi, essendo $\theta$ e $\Theta$ compresi fra $0^{\circ}$ e $180^{\circ}$. Dai segni della $[1.16]$ e della $[1.17]$ si deduce il quadrante in cui carle $A$ e quindi il suo valore.

Dalla fig. 1.6 si può dedurre un altro elemento importante del punto $P$, vale a dire l'angolo $\psi$, o declinazione geomagnetica. Si ha in valore assoluto

$$
\begin{aligned}
& \frac{\sin \psi}{\sin \vartheta_{o}}=\frac{\sin \left|\lambda-\lambda_{o}\right|}{\sin \Theta} \\
& \sin \psi=\frac{\sin \left|\lambda-\lambda_{o}\right| \sin \vartheta_{o}}{\sin \Theta}
\end{aligned}
$$

All'angolo $\psi$ si attribuisce tuttavia un segno, secondo la stessa convenzione usata per la declinazione magnetica: esso è positivo quando dal punto $P$ il polo geomagnetico è visto a est del polo geografico, negativo nel caso contrario (nel caso della fig. 1.6 esso è negativo). Ia [1.18] va allora sostituita dalla

$$
\sin \psi=\frac{\sin \left(\lambda_{0}-\lambda\right) \sin \vartheta_{0}}{\sin \theta}
$$

con una breve considerazione ci si convince facilmente, infatti, che con la $\left[1.18^{\prime}\right]$ si ottiene per $\sin \psi$ il segno attribuitogli dalla suddetta convenzione, pur di considerare la longitudine $\lambda$ variabile fra $0^{\circ} \mathrm{e}$ $360^{\circ}$, e contata positivamente verso est a partire dal meridiano di Greenwich. 


\subsection{ANOMALIE MAGNFTICHE.}

Tornanrlo al problema dell'anomalia magnetica in $P$ e quindi al calcolo del campo di dipolo in questo punto, osserviamo che la conoscenza della longitudine geomagnetica non i necessaria per le componenti $H$ e $Z$, perché per il campo di un dipolo esse dipendono solo dalla latitudine, come si è visto in (1.1):

$$
H=\frac{M \cos \Phi}{a^{3}} \quad, \quad Z=\frac{2 M \sin \Phi}{a^{3}} .
$$

Prendiamo, ad esempio, come punto P l'Osservatorio Geomagnetico di L'Aquila $\left(\varphi=42^{\circ} 23^{\prime} \mathrm{N}, \lambda=13^{\circ} 19^{\prime} \mathrm{E}\right)$. All'epoca 1965,0 si aveva: $H=23627 \gamma, Z=38532 \gamma, D=-1^{\circ} 15,9^{\prime}$. Assumendo come coordinate del polo geomagnetico quelle ottenute dall'analisi di Hurwitz e coll., che si riferisce alla stessa epoca $\left(\varphi_{0}=78^{\circ} 35^{\prime} \mathrm{N}, \lambda_{o}=69^{\circ} 49^{\prime} \mathrm{W}\right)$, dalla [1.15] si ottiene per $\Phi$ il valore di $42^{\circ}+2^{\prime} \mathrm{N}$ (alle nostre longiturlini la latituline geomagnetica è pressoché coincirlente con la latiturline geografica). Si hamno allora per $H$ e $Z$ del campo di dipolo i valori $H_{d}=22791 \gamma \quad(\mathrm{nT}), \quad Z_{d}=42073 \gamma \quad\left(\mathrm{nT}^{\mathrm{T}}\right)$; le anomalie sono quindi $A H \approx+840 \gamma(\mathrm{nT}), \Delta Z \approx-3540 \gamma(\mathrm{nT})$. Quanto alla declinazione, dalla $\left[1.18^{\prime}\right)$ si ricava per $w$ il valore di $-15,4^{\circ}$, per cui l'anomalia ì $\Delta I \approx+14,1^{\circ}$. E evislente l'influenza dell'anomalia asiatica, come si può vedere chiaramente dalla mappa di fig. 1.5.

Un modo semplice e significativo di indicare lo stato di anomalia di un punto rispetto al dipolo, che ci sarà utile nel seguito, è il calcolo rel cosirletto polo geomagnetico virtuale; ̀̀ questo il polo geomagnetico che risulterebbe dai valori della inclinazione e della declinazione effettivamente misurati nel punto, nella ipotesi che essi non siano anomali. Dalla $Z==H \operatorname{tg} I$ e dalle [1.1] si ottiene $\operatorname{tg} I=2 \operatorname{tg} \Phi$; da questa si alcola per il punto la latiturline greomagnetica virtuale $\Phi_{v}$. Dalla fig. 1.6 e dalla [1.18'], dove ora in luogo di $\psi$ si introrluca la declinazione effettiva $D$, si ha

$$
\begin{gathered}
\left.\sin \varphi_{o v}=\sin \Phi_{v} \sin \varphi+\cos \phi_{v} \cos \varphi \cos I\right) \\
\sin \left(\lambda_{v}-\lambda\right)=\frac{\left.\cos \Phi_{v} \sin I\right)}{\cos \varphi_{o v}} .
\end{gathered}
$$


Anche qui, per ottenere il valore dell'angolo $\left(\lambda_{o v}-\lambda\right)$, occorre conoscere il quadrante in cui esso si trova; ancora dalla fig. 1.6 si ricava

$$
\cos \left(\lambda_{o v}-\lambda\right)=\frac{\sin \Phi_{v}-\sin m \sin \varphi_{o v}}{\cos \varphi \cos \varphi_{o v}}
$$

Il quadrante si deduce dai segni di $\sin \left(\lambda_{o v}-\lambda_{\text {. }}\right)$ e di $\cos \left(\lambda_{\text {ov }}-\lambda\right)$.

Eseguendo i calcoli per L'Aquila per l'epoca 1965,0 $\left(I=58^{\circ 29}\right.$ '), si ottengono per il polo geomagnetico virtuale le coordinate

$$
\varphi_{o v}=86^{\circ} 40^{\prime} \mathrm{N}, \lambda_{v v}=210^{\circ} 25^{\prime} \mathrm{E} .
$$

In fig. 1.7 sono inclicate le posizioni del polo geomagnetico e del polo geomagnetico virtuale di L'Aquila.

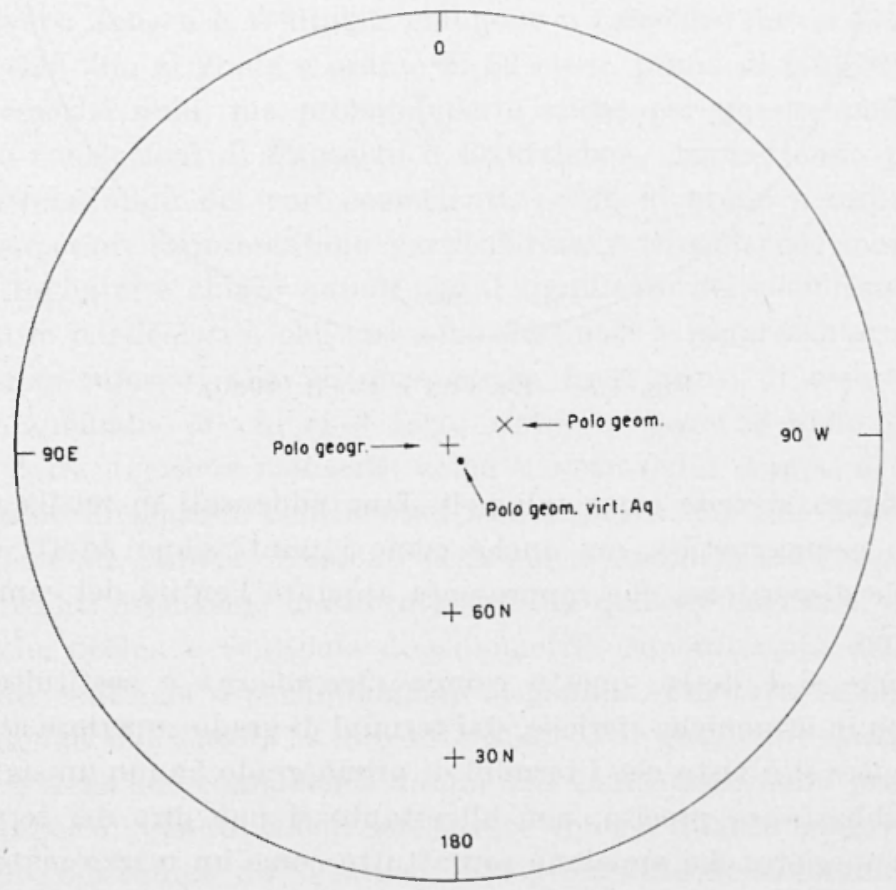

Fig. 1.7

Analogo procedimento si può applicare a ogni punto del globo, in particolare agli Osservatori (in cui le misure sono più sicure); la 
fig. 1.8 riporta i poli virtuali alcolati per 37 Osservatori all'epocab 1945.

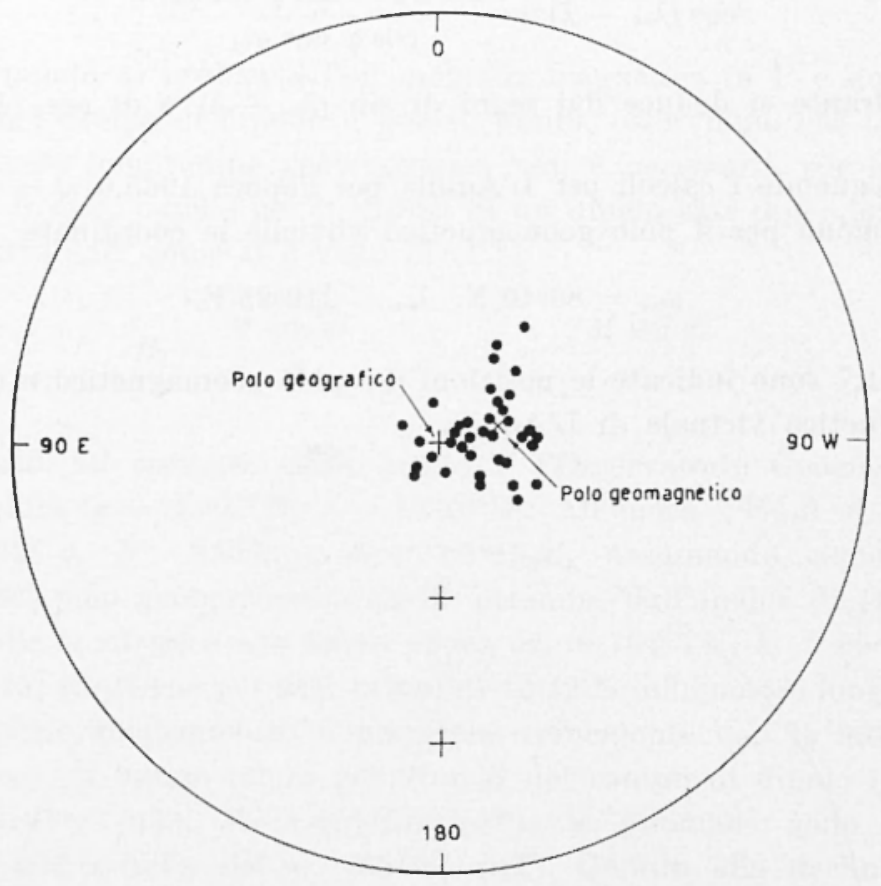

Fig. 1.8 - Da Cox e Doell (1960).

Dalla figura si verle come tali poli siano adrlensati in merlia attorno al polo geomagnetico, ma anche come i punti siano afietti da una notevole dispersione, che rappresenta appunto l'entità dlel eampo "irregolare s.

Come si ì rletto, questo campo "irregolare" i costituito, nello sviluppo in armoniche sferiche, dai termini di grarlo superiore al primo. Ma mentre si è visto che i termini di primo grarlo hanno un significato fisico abbastanza preciso, non altrettanto si può dire rlei termini di grarlo superiore, che appaiono soprattutto come un mezzo matematico per rappresentare le "irregolarità " del campo. Dal punto di vista fisico tutte queste irregolarità potrebbero essere spiegate dall'introduzione di semplici dipoli aggiuntivi al dipolo principale, come is stato messo in luce da MeNish (1940), secondo cui le principali caratteristiche del ampo irregolare possono venire rappresentate da 14 dipoli radiali 
situati a cirea $3000 \mathrm{~km}$ di profondita, ciò al limite tra il mantello e il nucleo terrestre (vedi (ap. 3). Va però osservato che, per la teoria del potenziale, le combinazioni di dipoli (o di correnti elettriche) che danno luogo a una determinata configurazione del campo sono in numero matematicamente infinito, e quindi i 14 dipoli di McNish rappresentano solo una soluzione possibile, forse probabile, ma non certa.

Si pone ovviamente il problema del grado e dell'ordine dei coefficienti di Gauss fino ai quali sarebbe opportuno estendere le analisi per rappresentare il più correttamente possibile il campo. Aleune delle analisi più note, ad es. quella di Vestine e coll. (1947), ginngono fino a $n, m=6$; Fanselau e Kautzleben (1958) hamno completato l'analisi dei dati usati da Vestine e coll. estendenclola fino a $n, m=15$, ma essi affermano che $\mathrm{i}$ termini di grato e ordine superiore a 6 sono in realtà inutili, riflettendo soprattutto la imprecisione dei dati. Con dati diversi Jensen e Whitaker giungono a calcolare fino a 512 coefficienti, ciò fino al grado e ordine di 22 circa, prima di giungere a valori pressoché nulli; ma probabilmente anche per questa analisi valgono le conclusioni di Fanselau e Kautzleben. Ammettendo per ipotesi l'attendibilità dei vari coefficienti, quelli di grado e ordine man mano superiori rappresentano particolarità, o irregolarità, man mano più dettagliate; è chiaro quindi che il significato dei coefficienti cessa quando le particolarità che essi sono destinati a rappresentare hanno dimensioni inferiori alla distanza merlia fra i punti di osservazione.

te anomalie di cui si ̀̀ fatto cenno a pag. 28 sono generalmente dette anomalie regionali; come si verle dalla mappa di fig. 1.5 esse hanno dimensioni continentali e intensità dell'ordine delle decine di migliaia di gamma. Esistono però anche anomalie, dette anomalie locali, aventi estensione molto inferiore (da qualche centinaio di metri a qualche decina o centinaia di chilometri), con intensità dell'ordine di alcune centinaia o poche migliaia di gamma. Per tutte le anomalie, sia regionali che locali, la loro estensione e il gracliente spaziale del campo e delle sue componenti danno una indicazione sulla profondità delle sorgenti: quanto meno estese esse sono e quanto maggiore è il gradiente del campo tanto minore è la profondità della sorgente. Alla luce di questa considerazione è interessante il risultato ottenuto da Alldredge e coll. (1963) dallo studio di un profilo magnetico attorno al mondo eseguito dall'aereo, nella fascia di latitudine compresa fra $12^{\circ} \mathrm{N}$ e $52^{\circ} \mathrm{N}$; gli $\Lambda \Lambda$. trovano che le anomalie si possono clividere in 
due gruppi, quelle di estensione di migliaia di chilometri e quelle di estensione delle centinaia e anche meno, con un'ampia lacuna fra $i$ due gruppi. In altre parole, anche le sorgenti delle anomalie magnetiche si possono dividere in due gruppi: quelle profonde aleune migliaia di (chilometri (nucleo terrestre) e quelle limitate alla crosta; vi ì quindi un largo intervallo di profondita privo di sorgenti di anomalie. Vedremo in seguito il significato di tale conchusione. Aggiungiamo invece ora che da varie considerazioni sull'applicazione dell'analisi armonica ai loro dati sperimentali gli $A A$. deducono che non occorre estendere un'analisi in armoniche sferiche al di la di $n, m-10$ per rappresentare il campo geomagnetico nei limiti di precisione richiesti dalle normali carte magnetiche planetarie. Ina ciò, tenendo anche conto delle conclusioni di Fanselau e Kautzleben e delle considerazioni fatte a pag. 21 sull'effetto del troncamento della serie di armoniche sferiche, si può grosso morlo aftermare che un'analisi estesa fino a $n, m=8$ i necessaria e sufficiente.

\subsection{LAA VARILZIONE SECOLARE.}

Se si rletraggono dai valori rlelle componenti del campo in un punto della Terra le variazioni dovute all'andamento regolare diurno e quelle dovute a pertubazioni o tempeste, si trova che il campo geomagnetico non è ancora costante nel tempo. Si ha invece una variazione lenta ma continua degli elementi del campo, detta variazione secolare. Un esempio può essere dato dai risultati dell'Osservatorio di L'Aquila: calcolando i valori medi annui dei tre elementi $D, H, Z$ si trova che dal 1960 al 1969 essi sono variati di circa $+33^{\prime}$ per la declinazione, con una media di $+3,6^{\prime}$ all'anno, di $+173 \gamma$ (nT) in $H$, con una merlia di $+19,2 \gamma$ (nT) all'anno, e di $+166 \gamma$ (nT) in $Z$, con una merlia di $+18,4 \gamma$ (nT) all'anno. Seguendo il suggerimento di Gaibas-Pnertas, possiamo calcolare la variazione dell'elemento $G=\left(H^{2}+\frac{Z^{2} \backslash 1 / 2}{4}\right)^{\prime \prime}=\frac{M}{a^{3}} ; G$ rappresenta l'intensitì del campo equatoriale che verrebbe prorlotto da un dipolo centrale di momento $\boldsymbol{M}$, il quale nel punto in esame desse luogo alle due componenti di valore $H$ e $Z$. 
L'andamento di to a LiAquila nei dlieci anni considerati è rappresentato in fig. 1.9.

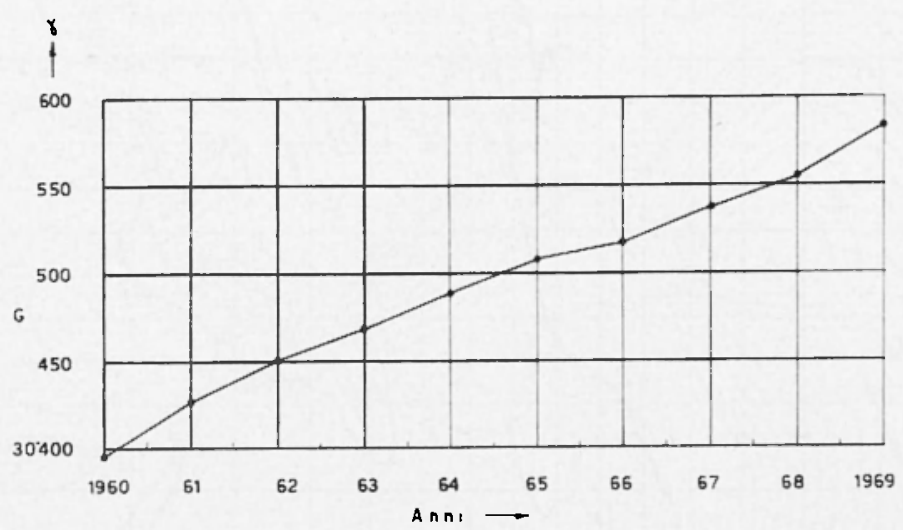

Fig. 1.9

Nella fig. 1.10 riportiamo l'andamento di $t r$ in vari Osservatori suddivisi in varie zone geografiche, per periodi molto più lunghi di quello che è possibile consilerare per L'Aquila; è evirlente che esso varia nettamente da zona a zona, presentando un continuo aumento in qualehe Osservatorio, una continua diminuzione in altri, e un andamento oscillatorio in altri ancora.

Il risultato di Gaibas-Puertas di fig. 1.10 fa pensare alla variazione secolare come arl un fenomeno regionale e non planetario. Tale conclusione verrebbe confermata dalla mappa delle isopore, cioè delle linee che uniscono i punti del globo che presentano la stessa variazione secolare espressa in gamma/anno (la fig. 1.11 si riferisce alla componente verticale all'epoca 1942,5 ). E evidente da tale mappa che le isopore costituiscono dei sistemi di curve di forma pressappoco ovale, distribuiti in morlo del tutto indipendente dalle configurazioni geografiche. Il sistema di isopore che interessa l'Italia è quello con centro a sul-ovest del mar Caspio, dove $Z$ aumenta di cirea $100 \gamma(\mathrm{nT})$ all'anno.

Ma occorre esaminare più a fondo questa "regionalità " del fenomeno. Nella Tab. 1 appare chiara una continua diminuzione del momento di dipolo.$I I$; non è possibile attribuire questa diminuzione soltanto ad un effetto apparente dovuto al maggior numero e alla migliore distribuzione delle stazioni nei tempi più recenti. $A$ smentire questa ipotesi basterebbe ad esempio il fatto che l'analisi di Fritsche 

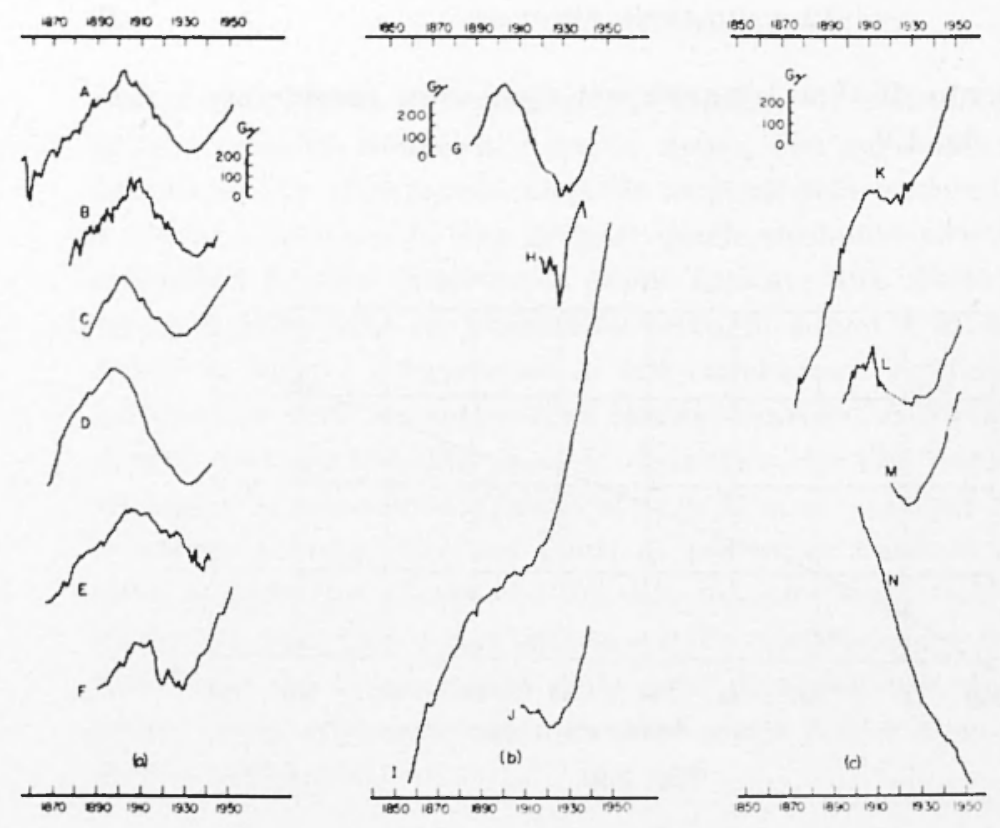

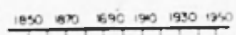
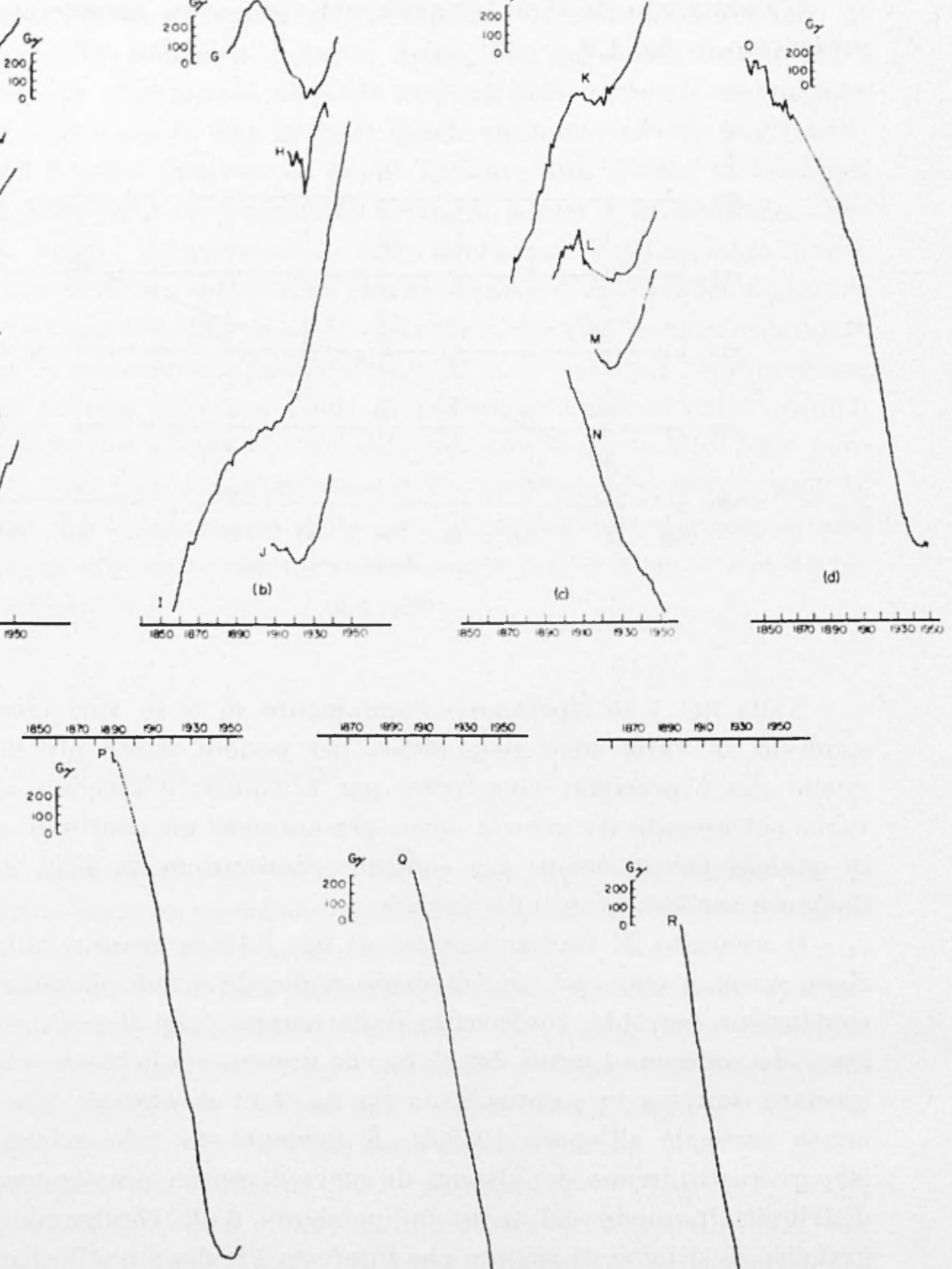

(e)
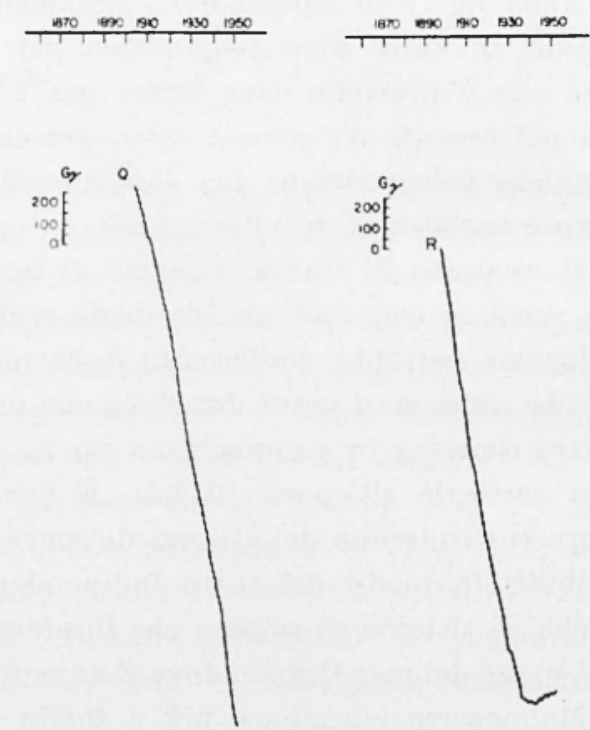

(f)
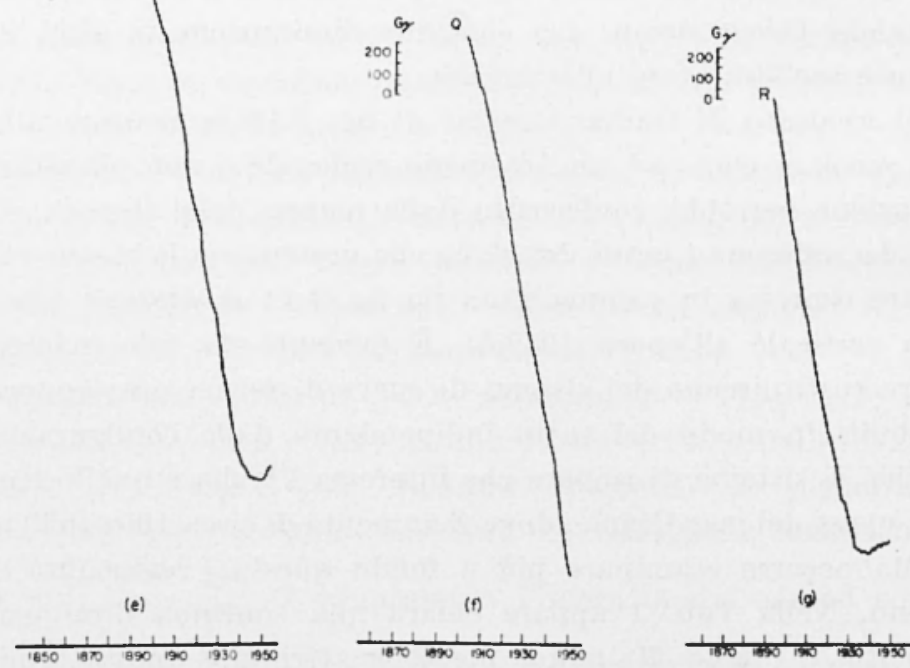

(9)

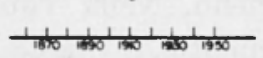

Fig. 1.10 - (a) Europa; (b) Asia centro-meridionale; (c) Estremo Oriente e Pacifico centro-meridionale; (d) Canalia; (c) U.S.A. Orientali; (f) Argentina; (g) Oceano Indiano (da Gaibar-Puertas 1953). 


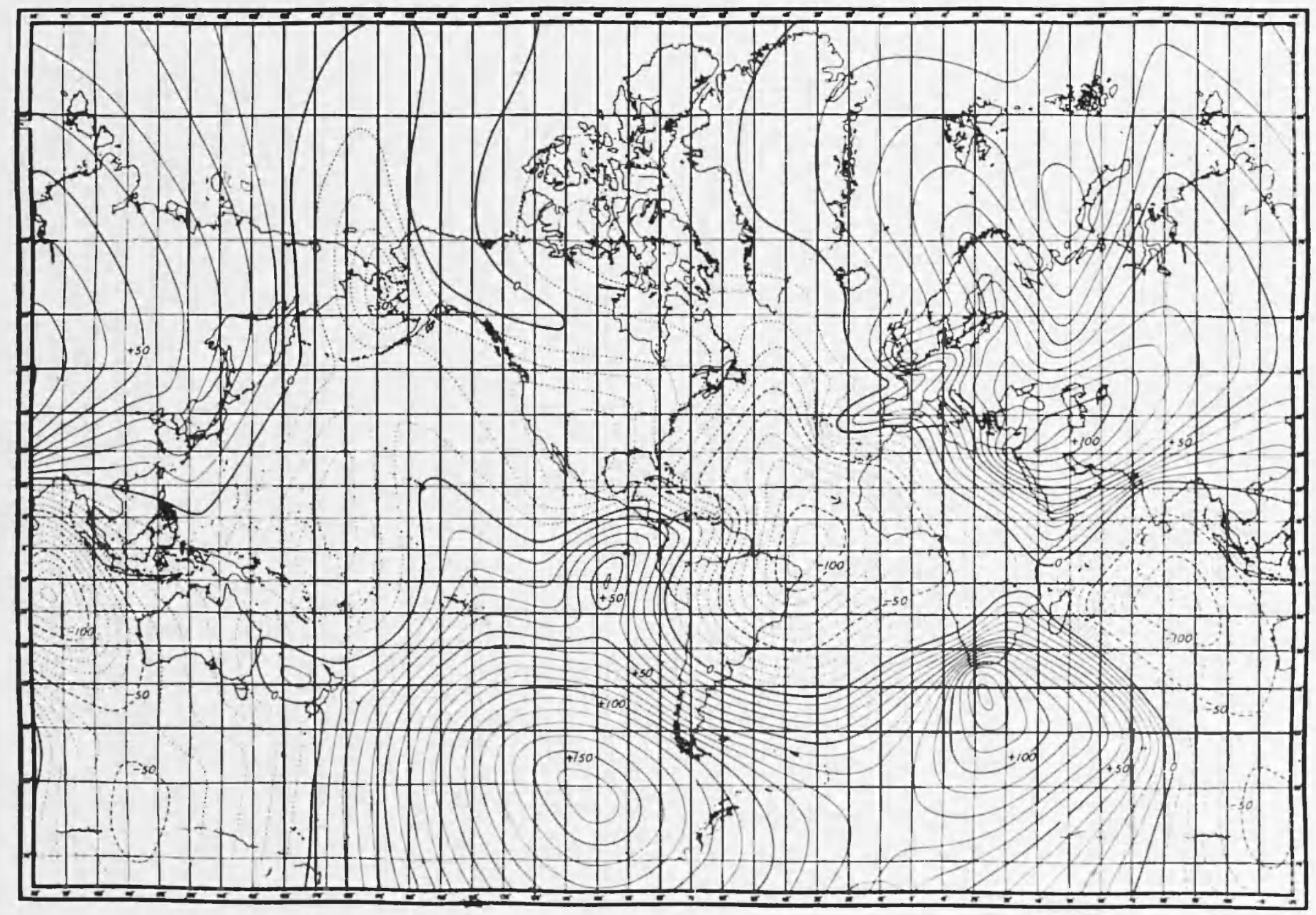

Fig. 1.11 - Isopore della componente verticale all'epoca 1942.5 (da Vestine e coll. 1947). 
(1885), basata su 408 stazioni, da $M=8,347 \cdot 10^{25}$ u.e.m. $\left(8,347 \cdot 10^{22}\right.$ $\mathrm{Am}^{2}$ ), mentre l'analisi di Schmiclt per lo stesso anno, basata su 1800 punti, dà $M=8,375.10^{25}$ u.e.m. $\left(8,375.10^{22} \mathrm{Am}^{2}\right)$, cioc̀ un valore maggiore.

Ammessa allora come reale la diminuzione di $M$, essa deve produrre evirlentemente un efletto planetario; ed è interessante notare

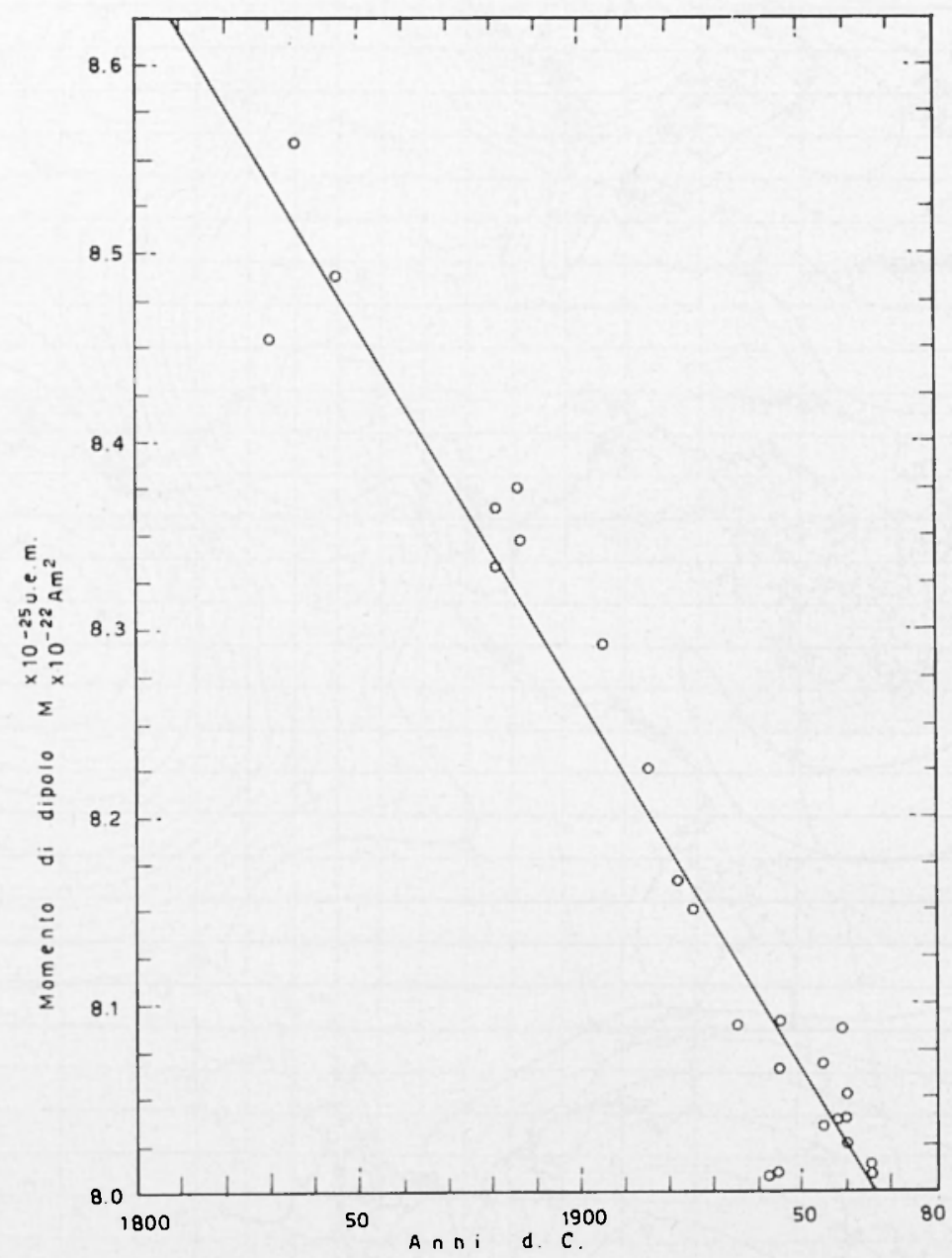

Fig. 1.12 - Da McDonald e Gunst (1967).

(fig. 1.12) che negli ultimi 130 anni la diminuzione è stata lineare, e potrebbe essere rappresentata merliante l'espressione 


$$
M=(-0,0039508 t+15,769) \cdot 10^{25} \text { u.e.m. }
$$

dove $t \dot{e}$ il tempo espresso in anni $A . D$. I probabilmente temerario estrapolare quest'andamento anche per il futuro: comunque ciò facendo si troverebbe l'anmulamento del momento di dipolo nell'anno 3991 .

Per quanto riguarda la direzione dell'asse del dipolo, dalla Tab. 1 appare evidente che la sua inclinazione rispetto all'asse terrestre ì rimasta, nell'intervallo di tempo coperto dalle analisi della tabella, costante, ¿̀ cioè rimasta costante la colatitudine del polo geomagnetico. Quanto alla longitudine, secondo McDonald e Gumst si può valutare uno spostamento a ovest dell'asse del dipolo con velocita media di $0,0418^{\circ}$ all'anno. Altri Autori, come Nagata (1965), danno un valore di $0,06^{\circ}$ all'anno per l'epoca 1960 . Secondo quest'ultimo Autore, inoltre, il dipolo centrale si sta spostando verso nord alla velocita di circa $2 \mathrm{~km} / \mathrm{annno.}$

Le variazioni del dipolo centrale devono dar luogo, come si is detto, a un effetto planetario; tuttavia dalla grancle varietà di forma e di intensita delle variazioni riportate in fig. 1.10 risulta chiaro che esse non bastano a spiegare la variazione secolare in un punto del globo.

Esiste però un altro fenomeno di carattere planetario che puó validamente contribuire alla spiegazione: tutto il campo "irregolare" (non dipolare) presenta una deriva verso ovest di circa $0,20 / a m n o$. E allora evidente che i diversi punti della Terra risentono una variazione secolare diversa a seconda della configurazione delle anomalie esistente in quei punti: il gradiente temporale delle singole componenti, ciò appunto la variazione secolare, dipende dal gradiente spaziale nei punti in esame e dalla velocità di spostamento dei sistemi di isoanomale. Ciò che ì importante chiarire ì quanto della variazione secolare osservata in un determinato punto della Terra sia dovuto a questa deriva occidentale e quanto a una variazione dellintensita del campo non dipolare. È facile valutare il contributo della deriva occidentale: una rotazione di tutto il campo non dipolare di $0,2^{\circ}$ all'anno fa passare in dieci anni il valore dell'anomalia presente in un punto all'istante $t=0$ al valore esistente, sempre all'istante $t=0$, in un punto di longitudine $2^{\circ}$ pin a est. Osservando la mappa del campo non dipolare di fig. 1.5, un calcolo piuttosto approssimativo ci permette di dedurre per l'Italia un aumento della componente verticale di circa 
$40 y$ (nT) all'anno; in realtà, come si è detto a pag. 36, l'aumento medio nell'intervallo $1960-1969$ ¿̀ stato di circa $20 \gamma$ (nT) all'anno. Si può quindi concludere che, oltre alla variazione secolare dovuta alla deriva occiclentale, esiste una diminuzione di circa $20 \gamma$ (n'T) all'anno nella componente verticale, il che potrebbe indicare un continuo intensificarsi della grande anomalia asiatica il cui bordo occidentale interessa l'Italia.

Questo esempio riguarlante la variazione secolare in Italia non is che una elementare applicazione di un metorlo generale usato da vari Autori, in particolar modo da Yukutake (1962), per cercare di valutare i due contributi alla variazione secolare del campo non dipolare. Chiamata $H$ una componente del campo magnetico terrestre, la variazione secolare $\partial H / \partial t$ in $u n$ determinato punto viene considerata come somma di due termini: il primo è $v \partial H / \partial \lambda$, dove $v$ è la velo(ità sulla superficie terrestre del eampo non dipolare dovuta alla deriva occidentale, il seconclo, espresso da $R(t)$, è il campo residuo, funzione del tempo, che risulta dalla clifferenza $\partial H / \partial t-v$ or $/ 0 \%$ e rappresenta le fluttuazioni in intensità del ampo non dipolare. Il metodo consiste nel trovare quel valore della velocità $v$ che rencla minimo il quarlrato del campo $R$ integrato, al esempio, lungo un intero parallelo. Se i valori della velocità $v$ cosi derlotti usando dati diversi di epoche diverse sono concordanti, si puó concludere che la deriva oceidentale è la principale causa della variazione secolare e allo stesso tempo si puó calcolare l'ordine di grandezza del campo residuo $R(t)$; se invece essi differiscono notevolmente fra loro, allora si dovrà decure che le fluttuazioni di intensità del campo non dipolare sono più importanti della deriva occirlentale. E appunto applicando questo metorlo o metodi simili che Yukutake e Bullard e coll. hanno stabilito che: a) la deriva occiontale fornisce il contributo più importante alla variazione secolare del campo non dipolare; b) la velocità $v$ è di $0,2^{\circ}$ all'anno (come abbiamo già accennato); $c$ ) le fluttuazioni di intensità del campo non dipolare sono dell'ordine di $10 \gamma$ (nT) all'anno; d) la configurazione del campo non clipolare ha una vita media di qualche centinaio di anni.

Per la determinazione di $v$ è stato usato anche un altro metodo. Riprendendo l'espressione [1.10] di 1.5 , possiamo serivere il potenziale $\mathrm{I}$ di origine interna alla Terra nella forma

$$
V=\imath \sum_{m=n}^{n}\left(\hat{r}_{m} \cos m \hat{\lambda}+H_{m} \sin m i\right)
$$




$$
\begin{aligned}
& \boldsymbol{r}_{m}=\sum_{n=1}^{\infty}\left(\frac{a}{r}\right)^{n+1} g_{n}^{m} P_{n}^{m}(\vartheta) \\
& H_{m}=\sum_{n=1}^{\infty}\left(\frac{a}{r}\right)^{n+1} h_{n}^{m} P_{n}^{m}(\vartheta)
\end{aligned}
$$

Lat [1.19] si può anche scrivere

$$
r=a \sum_{m=0}^{n} C_{m} \cos \left(m /-\varphi_{m}\right)
$$

con

$$
\begin{aligned}
& \boldsymbol{C}_{m}=\left(G_{m^{2}}^{2} \dashv-H_{m^{2}}\right)^{1 / 2} \\
& \operatorname{tg} \varphi_{m}=\frac{H_{m}}{G_{m}} .
\end{aligned}
$$

Calcolando le $r_{m}$ e $H_{m}$, ciò in altre parole i coefficienti $g_{n}^{\prime \prime}$ e $h_{1,}^{\prime \prime}$, in epoche diverse, si è trovato che le fasi $\varphi_{m}$ climinuiscono continuamente nel tempo; anche questo rivela una deriva verso ovest delle caratteristiche del campo non dipolare, e dalla diminuzione delle $\varphi_{m}$ si può dedurre, almeno in linea di principio, la velocità della deriva per le varie armoniche superficiali. Tenendo conto che le $\varphi_{m}$, per le [1.20], dipendono da $\%$, il metodo permette di calcolare la velocità di deriva in funzione rlella latitudine. Applicando questo metodo, mentre si ì confermato l'ordine di grandezza della velocità di deriva, non è stato peró ancora chiarito l'ultimo problema accennato, e cioè se questa velocita di spostamento sia uguale su tutta la Terra o se essa dipenda dalla latitudine, o se infine vari casualmente da regione a regione; sotto questo punto di vista vi sono risultati discordanti, poiché secondo qualche Autore (Yukutake 1967) la velocità di deriva aumenta sensibilmente con l'aumentare della latitudine, mentre secondo altri (Witham 1958) in Canada la deriva a ovest è circa un terzo della media mondiale.

Un fatto sperimentale molto importante per la interpretazione della variazione secolare è la scoperta (Vestine 1953) che alle piccole fluttuazioni della velocità di rotazione terrestre, dedotte per via astronomica, corrispondono fluttuazioni nella deriva occidentale del campo magnetico. Jalla fig. 1.13, dove ai dati magnetici viene dato maggior peso, per ovvie ragioni, dopo il 1900, si nota chiaramente che fra il 1910 e il 1930 an una diminuzione del periorlo di rotazione terrestre 
di circa 1,5 sece (cioe ad una rotazione verso est della crosta terrestre più rapicla del normale, corrisponde un aumento della velocità di deriva occidentale del campo.

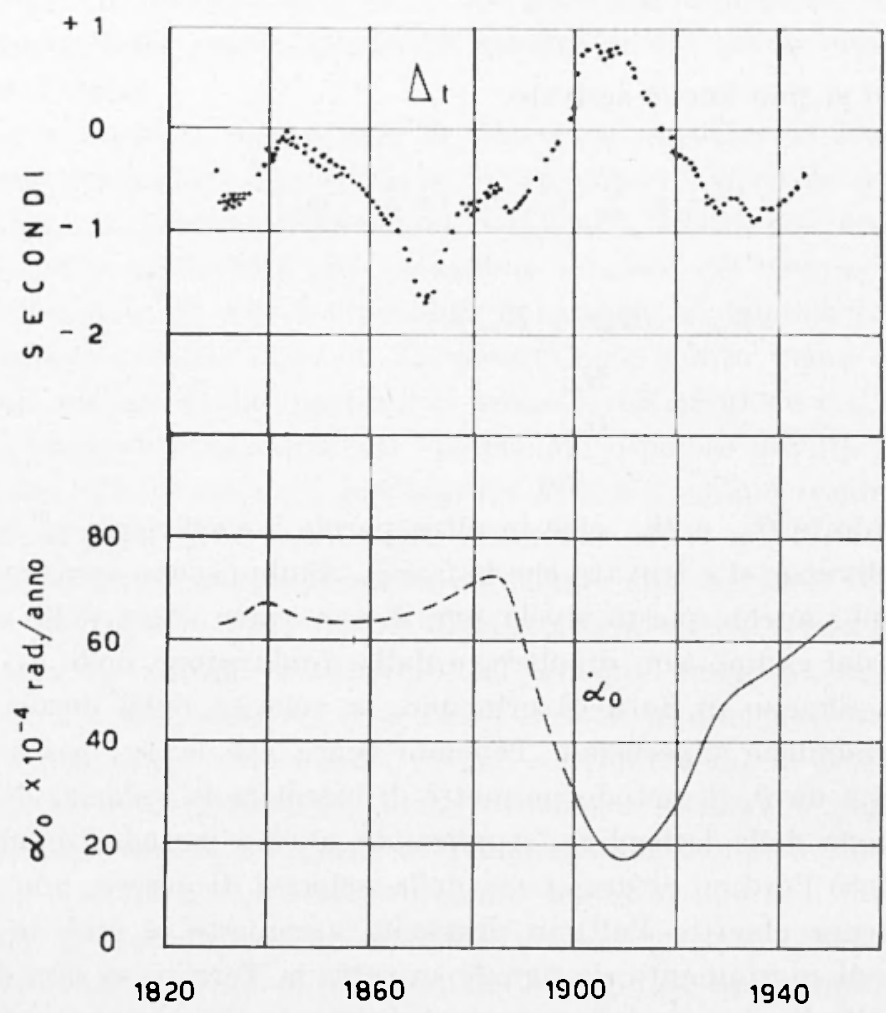

Fig. 1.13 - $\Lambda_{\ell}$ : variazioni nel periodo di rotazione terrestre: $a_{0}$ deriva occidentale (da Vestine 1953).

Un altro fatto piuttosto interessante i che nella zona rlel Pacilico la variazione secolare è nettamente inferiore (di circa un fattore 3 ) a quella che in media si riscontra nel resto della Terra. Cio potrebbe essere spiegato, alla luce della deriva occirlentale, dal fatto che i gradienti spaziali del campo anomalo sono in questa zona piuttosto bassi, in altre parole che la zona del Pacifico non presenta anomalie molto accentuate o, ancora, che il campo non dipolare è quasi assente. Tale spiegazione non è tuttavia sufficiente, poiché risulta dagli sturli paleomagnetici che anche mezzo milione di anni fa la variazione secolare 
nel Pacifico presentava lo stesso carattere, e rimarebbe quindi da risolvere il problema della permanente assenza da questa zona di grandi anomalie.

Quest'ultimo accenno ei pone di fronte a una questione importante: noi conosciamo il campo magnetico terrestre solo dalle osservazioni eseguite negli ultimi quattro secoli, ma 400 anni rappresentano un istante brevissimo nella storia della Terra. Le conoscenze relative a questo breve istante non sono certo sufficienti a permetterei di avanzare delle fondate ipotesi sull'origine del campo; dobbiamo quindi cercare un mezzo per conoscere qualche cosa sulle caratteristiche di esso durante le ere geologiche. Questo mezzo ci is fornito dal magnetismo delle rocce, o paleomagnetismo. 